University of South Florida

DIGITAL COMMONS

Digital Commons @ University of

@ UNIVERSITY OF SOUTH FLORIDA

South Florida

2014

\title{
On the Ability of Global Sea Level Reconstructions to Determine Trends and Variability
}

\author{
F. M. Calafat \\ University of South Florida \\ D. P. Chambers \\ University of South Florida, donc@usf.edu \\ M. N. Tsimplis \\ National Oceanography Centre, Southampton
}

Follow this and additional works at: https://digitalcommons.usf.edu/msc_facpub

Part of the Life Sciences Commons

\section{Scholar Commons Citation}

Calafat, F. M.; Chambers, D. P.; and Tsimplis, M. N., "On the Ability of Global Sea Level Reconstructions to Determine Trends and Variability" (2014). Marine Science Faculty Publications. 1411.

https://digitalcommons.usf.edu/msc_facpub/1411

This Article is brought to you for free and open access by the College of Marine Science at Digital Commons @ University of South Florida. It has been accepted for inclusion in Marine Science Faculty Publications by an authorized administrator of Digital Commons @ University of South Florida. For more information, please contact digitalcommons@usf.edu. 


\title{
Journal of Geophysical Research: Oceans
}

\section{RESEARCH ARTICLE On the ability of global sea level reconstructions to determine 10.1002/2013JC009298 trends and variability}

Key Points:

- Variability is better captured if the EOFO is not used

- The reconstruction method with the EOFO does not use global information

- The reconstructed GMSL without the EOFO is correlated with hydrology changes

Correspondence to:

F. M. Calafat,

Francisco.Calafat@noc.ac.uk

Citation:

Calafat, F. M., D. P. Chambers, and M. N. Tsimplis (2014), On the ability of global sea level reconstructions to determine trends and variability, $J$. Geophys. Res. Oceans, 119, 1572-1592, doi:10.1002/2013JC009298.

\author{
F. M. Calafat ${ }^{1,2}$, D. P. Chambers ${ }^{1}$, and M. N. Tsimplis ${ }^{2}$ \\ ${ }^{1}$ College of Marine Science, University of South Florida, St. Petersburg, Florida, USA, ${ }^{2}$ National Oceanography Centre, \\ Southampton, UK
}

\begin{abstract}
We investigate how well methods based on empirical orthogonal functions (EOFs) can reconstruct global mean sea level (GMSL). We first explore the analytical solution of the method and then perform a series of numerical experiments using modeled data. In addition, we present a new GMSL reconstruction for the period 1900-2011 computed both with and without a spatially uniform EOF (EOF0). The method without the EOF0 uses global information, which leads to a better reconstruction of the variability, though with some underestimation. The trend, however, is not captured, which motivates the use of the EOFO. When the EOFO is used the method reduces to the generalized weighted mean with regularization of altimetry records at tide-gauge locations, and thus it uses no global information. This results in a poor reconstruction of the variability. Although the trend is better captured (biases smaller than $\pm 25 \%$ ) with the EOF0, using the covariance matrix of deseasonalized monthly time series as the basis for determining the contribution of each tide gauge to the trend is dubious because it assumes that the interannual variability and the trend are driven by the same mechanisms. A significant fraction of the interannual to decadal variability ( $\sim 4 \mathrm{~mm}$ peak-to-peak and $\sim 2 \mathrm{~mm}$ standard error) in the new GMSL reconstruction without the EOF0 is consistent with land hydrology changes associated with the El Niño-Southern Oscillation (ENSO). When the EOFO is used, we find no correlation with either the ENSO or land hydrology changes, and decadal fluctuations are $\sim 5$ times greater.
\end{abstract}

\section{Introduction}

Sea level is one of the most useful variables for monitoring climate change and variability as it reflects both mass addition from melting ice sheets and heat content increase in the oceans. At the same time, it is a notoriously difficult variable to explain because it is a function of all physical and dynamic processes occurring within and among various components of the climate system, including air-sea heat and freshwater fluxes, changes in winds and ocean circulation, melting of water from ice sheets and glaciers, and responses of the solid earth to the redistribution of water mass. Over the past decades, substantial efforts have been devoted to better understand how each of these processes have contributed to sea level changes in the past with the aim of producing better projections of future sea level rise. This, however, has proved to be a complex task because, in addition to the several factors affecting sea level, considerable regional variability occurring over a wide range of temporal scales has been found.

In principle, resolving the problem would require the use of accurate measurements of sea level as well as the contributing parameters with good spatial and temporal coverage. What we have instead is a handful of tide gauges that provide sea level measurements for 100 years or longer, predominantly located on continental boundaries around Europe and North America. The number of suitable tide-gauge records increases gradually with time, especially after 1950 when measurements from islands in the middle of the oceans and in the Southern hemisphere become increasingly available (Figure 1). However, the first nearly global measurements of sea level were not available until the late 1980s with the advent of routine satellite altimeter measurements, and these were not precise enough until 1992 with the launch of the TOPEX/Poseidon mission. Note, however, that even these measurements do not cover the oceans fully and oceanic regions further north/south of around $66^{\circ} \mathrm{N} / 66^{\circ} \mathrm{S}$ are not sampled. Notably, the altimetric measurements do not extend to the coast but stop being useful at distances around $30 \mathrm{~km}$ from the coast.

Despite the lack of measurements, which is by no means unique to sea level research, several efforts have been made to synthesize the available measurements to produce a global sea level average. Earlier efforts 


\section{QAGU Journal of Geophysical Research: Oceans}

(a)

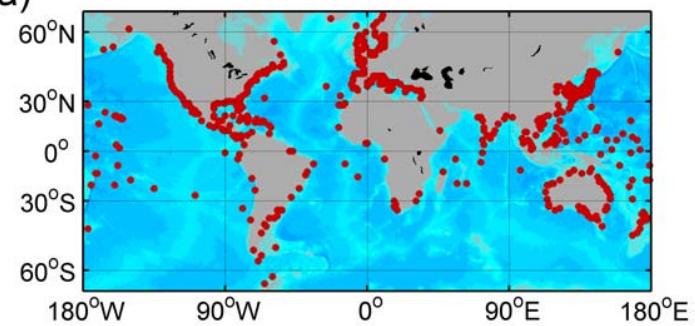

(b)

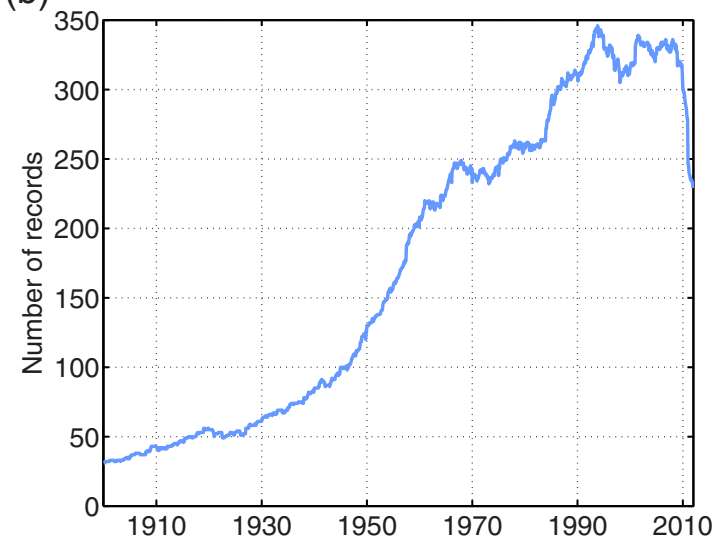

Figure 1. (a) The location of all composite tide-gauge records used in this study. (b) The number of composite tide gauge records over time (1900-2011). were based on averaging of tide gauges per region or selective averaging of the most reliable tide gauges. The development of altimetric measurements with near-global coverage created the opportunity to combine the relationship between tide gauges and altimetry over the common period of operation and produce reconstructions of sea level based on the spatial patterns evidenced by the altimetric measurements with the variability from long tide-gauge records [Chambers et al., 2002; Church et al., 2004; Church and White, 2011 (hereinafter CW2011); Berge-Nguyen et al., 2008; Calafat and Gomis, 2009; Llovel et al., 2009; Calafat and Jorda, 2011; Hamlington et al., 2011, 2012; Ray and Douglas, 2011; Meyssignac et al., 2012]. The most widely used technique to reconstruct sea level is the reduced space optimal interpolation (RSOI) described by Kaplan et al. [1997, 2000]. This technique was originally developed for the purpose of filling gaps to atmospheric observational fields like sea level atmospheric pressure. However the technique can, at least numerically, be extended to any sparse field. For sea level, the technique involves the calculation of empirical orthogonal functions (EOFs) from a spatially dense data set (e.g., satellite altimetry), which are then fitted, in a weighted least square

sense, to a set of tide-gauge records. To account for uniform sea level changes, Church et al. [2004] modified the technique by adding a spatially uniform EOF (also called EOF0) to the EOF basis used in their reconstruction. Since then, most EOF reconstructions have been calculated with the addition of the EOF0.

While sea level reconstructions have been routinely used to determine past sea level variability, to date little quantitative research has been done on the reliability of the sea level reconstructions, especially for determining interannual to multidecadal variability in global mean sea level (GMSL). All GMSL reconstructions exhibit variability at multidecadal ( $>30$ year) periods, but all differ significantly, and it is not clear whether the variability is truly a global variation or reflects sampling of larger regional variability [e.g., Chambers et al., 2012]. Our aim here is to assess how well the GMSL variability can be reconstructed using the RSOI method and to explore the various factors that affect the performance of the method, including the use of the EOF0.

There are various factors that contribute to errors in the EOF-based sea level reconstructions. First, the RSOI assumes that the EOFs calculated in the altimetry period are stationary in time. However, significant decadal and multidecadal sea level variability observed in many tide-gauge records around the world [Firing et al., 2004; Miller and Douglas, 2007; Merrifield, 2011; Calafat et al., 2012; Chambers et al., 2012; Marcos et al., 2012; Calafat et al., 2013; Dangendorf et al., 2013; Wahl et al., 2013] indicates that the spatial patterns of sea level variability fluctuate significantly at time scales much longer than those resolved by the altimetry data. A potential solution to this issue is the use of EOFs calculated from numerical models [Llovel et al., 2009; Meyssignac et al., 2012]. However, using patterns from a model assumes the model accurately represents patterns of sea level variability in the past, which is difficult to assess.

Additional sources of uncertainty are the poor spatial sampling of the tide-gauge data set, its uneven distribution around the world, and the way in which the distribution changes over time, all of which can lead to biased estimates of the GMSL. The method in essence assumes that the changes available at a number of tide gauges, even if these are $<10$, or spatially restricted, have information for the EOFs globally. This, of course, implies that the coastal information available at tide gauges is related to what happens in the open 
ocean. This assumption may be reasonable when one considers GMSL changes of the order of tens of metres over thousand of years. However, the variability at tide gauges is in many regions uncorrelated with the variability in the open ocean on seasonal [Vinogradov and Ponte, 2010] to decadal [Bingham and Hughes, 2012; Calafat et al., 2012] timescales, and hence this assumption may not be true. Physical factors contributing to the differences between coastal and deep ocean sea level are strong boundary currents, wind-driven upwelling/ downwelling, propagation of coastally trapped waves, complex topography, and sharp gradients in atmospheric surface fluxes at the land-ocean boundary, among others. An additional shortcoming is that, in some regions, the sea level variability at tide gauges is largely driven by coastal and shelf-sea processes that are not captured by altimetry. This results in significant differences between tide gauges and nearby altimetry.

To date, the only serious published attempt (to the best of our knowledge) to assess the reliability of the GMSL computed from EOF-based reconstructions is that of Christiansen et al. [2010] (hereinafter C2010). C2010 used an ensemble of surrogate sea level fields and synthetic tide gauges based on a climate model simulation to quantify the performance of various EOF-based methods in reconstructing the GMSL. They found that including the EOFO improved the skill of the RSOI method in reconstructing both the trend and the interannual variability. According to their results, the RSOI method with the EOFO could reconstruct the GMSL trend with a negative bias of $<10 \%$ and the interannual variability with correlations of up to 0.5 . Nevertheless, none of the interpolation methods was better, as regards the reconstruction of the long-term trend, than a simple average of the tide gauges. They also found that the magnitude of the variability was overestimated by all methods.

The study of C2010 was an important first step to help us understand the strengths and weaknesses of the GMSL reconstructions. However, as we will show, some of their results differ from what is found in GMSL reconstructions derived from observations, which suggests that some of their conclusions may not apply to reality. A possible explanation for the discrepancies could be that C2010 used an ensemble of surrogates based on an ocean model with a spatial resolution of about $5^{\circ}$ (longitude) $\times 4^{\circ}$ (latitude) with only 1850 ocean grid points. This spatial resolution is insufficient to resolve the complex shelf-sea and coastal processes which control the sea level variability at the tide gauges. Another possibility could be that $\mathrm{C} 2010$ used synthetic tide gauges obtained from the model grid points. As a result, these are consistent with the coastal sea level from the model, a similarity that does not hold when tide gauges are compared with nearby altimetry. In addition to this, the model used by C2010 did not account for the contribution of continental ice loss/gain to sea level. This contribution is not spatially uniform [Riva et al., 2010] and dominates the century-scale trend in GMSL [Cazenave and Llovel, 2010], and thus ignoring it may lead to biased results with regards to the reconstruction of the long-term trend.

It should be noted also that the EOFO was primarily included so that the secular trend could be captured, and thus, in principle, one would expect the variability described by the EOF basis to be better captured without the EOF0. However, C2010 have suggested that the phase of the variability is better captured with the EOF0 rather than without it. As GMSL reconstructions are routinely used to explore not only the trend but also accelerations and multidecadal variability, which are both affected by the reconstructed variability, it is necessary to implement a complete assessment of the fidelity of the variability in GMSL

reconstructions.

Our aim here is to assess the reliability of the reconstructed variability by exploring the analytical solution of the RSOI and performing a series of numerical experiments using both sea level observations and modeled data. Section 2 describes the data used in the numerical experiments and the statistical parameters used to assess the fidelity of the reconstruction. In section 3, we present a theoretical analysis of the RSOI solution. In particular, we rewrite the analytical solution for the RSOI in a notation that facilitates the interpretation of the numerical results presented later in the paper and brings out the strengths and weaknesses of the RSOI in reconstructing the GMSL. The results of the numerical experiments are presented in section 4 . We use the ensemble approach described by C2010, but with some modifications. The model used here has a much higher spatial resolution $\left(1^{\circ} \times 1^{\circ}\right)$ than that used by $\mathrm{C} 2010$, which, in principle, provides a better resolution of the processes controlling coastal sea level variability. In addition, our model accounts for the contribution of continental ice loss/gain and land hydrology to sea level. A new GMSL reconstruction with and without the EOFO for the period 1900-2011 is presented in section 5. We compare our GMSL reconstruction with other previously published reconstructions and also with various climate indices and for different timescales. Finally, section 6 summarizes and discusses the main results of the paper. 


\section{Data and Methods}

\subsection{Tide-Gauge Data}

In this study, we use tide-gauge records of monthly averaged time series of sea level from the Revised Local Reference (RLR) data archive of the Permanent Service for Mean Sea Level (PSMSL) [Woodworth and Player, 2003]. The RLR data set was downloaded in January 2013 and contains 1364 stations of varying length and quality. The selection of the tide-gauge stations used in the reconstruction is made following similar criteria as those by Church et al. [2004]. First, monthly values that are flagged by the PSMSL are rejected. Note that the PSMSL performs quality checks on the tide-gauge data and assigns a quality flag to each station (" $\mathrm{N}$ " or " $Y$," where " $Y$ " indicates that the entire station is flagged for attention) and also to each monthly value (" 000 " and "001", where a value of " 001 " indicates problems with the corresponding monthly value). In this first step, we reject monthly values that have been assigned a value of "001". There are 91 tide-gauge records in the RLR data set with at least one flagged monthly value. Second, gaps of 1-2 months are filled using cubic spline interpolation. Third, tide-gauge records are broken into separate sections whenever there is a month-to-month change $>250 \mathrm{~mm}$, but only when the number of such changes over the whole record does not exceed 10. The reason for using a threshold value is that when a tide-gauge record undergoes a considerable number of jumps $>250 \mathrm{~mm}$ (there are 48 records with 50 or more of such jumps over their time span), we consider such jumps to relate to regional sea level variability rather than to datum shifts. Although the choice of a threshold value of 10 is somewhat arbitrary, it has little effect on the results as most of the records having this problem (86\%) have 10 or less jumps $>250 \mathrm{~mm}$. Fourth, continuous sections shorter than 2 years are rejected. Fifth, tide-gauge records showing absolute linear trends $>10 \mathrm{~mm} / \mathrm{yr}$ are rejected (93 tide-gauge records are rejected). However the inclusion of the records with absolute trend values larger than $10 \mathrm{~mm} / \mathrm{yr}$ does not change the value of the GMSL trend significantly. Finally, all tide-gauge records are inspected visually to detect obvious outliers or datum shifts, paying particular attention to records that are flagged for attention by the PSMSL (i.e., those having a quality flag with a value of "Y"). Tide-gauge records that are too noisy or that are located at the mouth of a river are rejected during the visual inspection. After the selection procedure, a total of 892 records remained for use in the reconstruction.

The selected tide-gauge records are corrected for Glacial Isostatic Adjustment (GIA) using the solutions of the ICE-5G (VM2) model described in the work of Peltier [2004]. The GIA values are freely available on the PSMSL website. Tide-gauge records are also corrected for the effect of atmospheric pressure changes using the inverse barometer (IB) correction. The sea level pressure (SLP) data used to compute the IB correction are obtained from the near real-time update of the Hadley Centre Sea Level Pressure (HadSLP2r with reduced variance) data set [Allan and Ansell, 2006]. HadSLP2r combines marine and land pressure observations using a reduced-space optimal interpolation analysis and is available on a $5^{\circ}$ latitude-longitude grid from 1850 to present. Finally, since our focus is on interannual and longer timescales, the annual and semiannual cycles are computed and removed from each tide-gauge record by means of a harmonic analysis. Ideally, the seasonal cycle should be calculated over a common period for all records since it is known to be temporally variable [Marcos and Tsimplis, 2007], however the fact that tide-gauge records span different time periods makes that unfeasible. Hence, the seasonal cycle has been calculated over the entire period covered by each record.

Because the sea level reconstruction is produced on a $1^{\circ} \times 1^{\circ}$ grid we generate a set of composite records by finding the nearest grid point for each tide gauge, noting that tide gauges are averaged to produce a single time series where more than one tide gauge is found. Following Church et al. [2004], tide gauges whose nearest grid point is further than $250 \mathrm{~km}$ are rejected. The locations of all composite records together with the number of records over time are shown in Figure 1 (for the altimetry grid). Note that the number of tide gauges is much larger in the Northern Hemisphere than in the Southern Hemisphere. It is also interesting to note that the number of tide gauges decreases significantly as we move backward in time. In particular, the number of composite records is 31 in 1900, increases to 125 in 1950, and peaks at 346 in November 1992.

\subsection{Satellite Altimetry Data}

Gridded monthly mean sea level anomalies with a spatial resolution of $1^{\circ} \times 1^{\circ}$ for the period January 1993 to November 2012 were obtained from the CSIRO web site (http://www.cmar.csiro.au/sealevel/sI_data_ cmar.html). The gridded anomalies were produced using a combination of TOPEX/Poseidon, Jason-1, and 
Jason-2/OSTM altimetry missions with all geophysical corrections applied, including the IB and GIA corrections. The data used here had the seasonal cycle removed. The GIA correction applied to the data is described in CW2011.

\subsection{Climate Indices}

The variability associated with the El Niño-Southern Oscillation (ENSO) is represented by the Multivariate ENSO index (MEl, available at http://www.esrl.noaa.gov/psd/enso/). The MEl index is computed as the first unrotated PC of six observed variables (SLP, zonal and meridional components of the surface wind, sea surface temperature, surface air temperature, and total cloudiness fraction of the sky) combined over the tropical Pacific [Wolter and Timlin, 1998] and spans the period 1950-2011. For the years before 1950, we use the Extended MEl index (MEl.ext) [Wolter and Timlin, 2011]. The Atlantic Multidecadal Oscillation (AMO, available at http://www.esrl.noaa.gov/psd/data/timeseries/AMO/) index is computed as the area weighted average of sea surface temperature over the North Atlantic $\left(0^{\circ} \mathrm{N}-70^{\circ} \mathrm{N}\right)$ [Enfield et al., 2001].

\subsection{Model Data}

Simulated sea level data are obtained from the Simple Ocean Data Assimilation (SODA) reanalysis version 2.2.4 [Carton and Giese, 2008; Giese and Ray, 2011]. SODA is based on the Parallel Ocean Program (POP) model with an average horizontal resolution of $0.25^{\circ} \times 0.4^{\circ}$ and 40 vertical levels unevenly distributed with a resolution varying from $10 \mathrm{~m}$ near the surface to $250 \mathrm{~m}$ at the bottom. The model covers the entire global oceans and spans the period 1871-2008. The model output is provided in the form of monthly mean fields mapped onto a regular global $0.5^{\circ} \times 0.5^{\circ}$ grid. For the vertical mixing a K-Profile Parameterization is used, while horizontal diffusion is modeled using a biharmonic mixing scheme. Rivers are included with climatological seasonal discharge. The atmospheric forcing is provided by the 20th Century Reanalysis [Compo et al., 2011]. SODA assimilates all available temperature (T) and salinity (S) observations from hydrographic stations, XBTs, MBTs, and floats. In particular, surface T data are obtained from the ICOADS 2.5 data set, while subsurface T and S data are obtained from the World Ocean Database 2009 (WOD09) [Boyer et al., 2009]. For specific details of the SODA assimilation system, the reader is referred to Carton and Giese [2008].

Because the model is formulated under the Boussinesq approximation, it conserves volume but not mass, and hence it does not properly represent GMSL changes due to the expansion/contraction of the water column (i.e., steric effects), or caused by the exchange of water mass between oceans and land-based reservoirs. A simple way to correct for the missing steric effects is to add, at each time step, a spatially uniform sea level signal computed as the averaged steric height changes (calculated from the model density fields) over the global oceans [Greatbatch, 1994; Ponte, 1999]. After this correction, the sea level from the model accounts for steric effects (both regional and global) and mass redistribution, but not for water mass exchange between oceans and land.

Continental ice loss (from glaciers and ice sheets) is an important contributor to long-term trends in GMSL [Cazenave and Llovel, 2010]. We incorporate a model of ice mass contribution to sea level using fingerprints of the sea level response to ice mass losses from glaciers, Greenland, and Antarctica, as computed by Riva et al. [2010]. This is provided on a $0.5^{\circ} \times 0.5^{\circ}$ grid, which is added to the model. The fingerprint represents secular sea level trends due to continental ice loss/gain and is based on direct observations from the Gravity Recovery and Climate Experiment (GRACE) satellites. It has been obtained by assuming an equilibrium ocean with homogenous density, and thus it is complementary to our ocean model. Note that this approach assumes that the spatial pattern of sea level trends associated with water mass exchange between land and oceans does not change over time, and thus it is the same for both the GRACE period and the period covered by SODA.

Changes in land hydrology can also induce significant changes in the GMSL, although these affect primarily the interannual and interdecadal variability. We use the Climate Prediction Center (CPC) global monthly soil moisture data set [Fan and van den Dool, 2004] to produce an estimate of this contribution, assuming that water mass exchanges between oceans and land act as a barotropic load on the ocean. The CPC soil moisture data set is provided on a regular $0.5^{\circ} \times 0.5^{\circ}$ grid and spans the period 1948-2012. The sea level changes associated with land hydrology changes are estimated as a spatially uniform but time varying sea level signal, $\eta_{h}(t)$, given by 


$$
\eta_{h}(t)=-\frac{1}{A_{\text {Ocean }}} \int_{A_{\text {Land }}} F d A
$$

where $A_{\text {Ocean }}$ and $A_{\text {Land }}$ are the surface area of the global oceans and the continents, respectively, and $F$ is the soil moisture (detrended). The signal $\eta_{h}(t)$ is, then, basically spread over the whole global oceans by adding it, at each time step, to the sea level fields from the model [Ponte, 1999].

Ideally, we would like $\eta_{h}(t)$ to span the whole period covered by the ocean model (1871-2008), but unfortunately the CPC soil moisture data start in 1948. To extend the time series $\eta_{h}(t)$ back in time to 1871, we use a phase-randomized Fourier-transform algorithm. The algorithm consists of Fourier transforming the original time series $\eta_{h}(t)$, randomize the phases by rotating them at each frequency by an independent uniform random number in the range $[0-2 \pi]$, and then invert the transform [Prichard and Theiler, 1994]. The resulting time series, $\tilde{\eta}_{h}(t)$, has the same length, power spectrum, and autocorrelation function as the original time series $\eta_{h}(t)$. We apply the algorithm 2 times to obtain two surrogate time series, $\tilde{\eta}_{h}^{1}(t)$ and $\tilde{\eta}_{h}^{2}(t)$, spanning the periods 1871-1882 and 1883-1947. Then, the original and the two surrogate time series are concatenated to form a single long time series spanning the period 1871-2008.

(a)

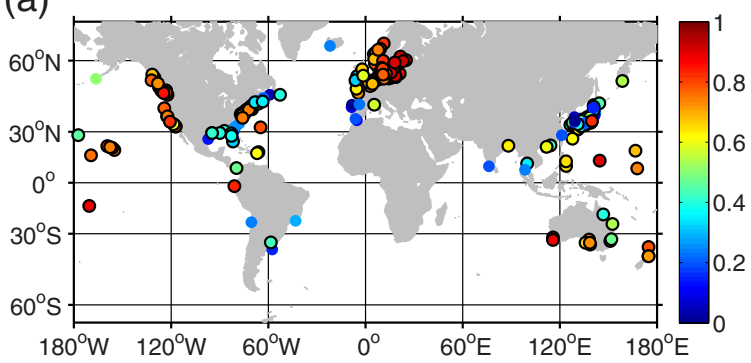

(b)

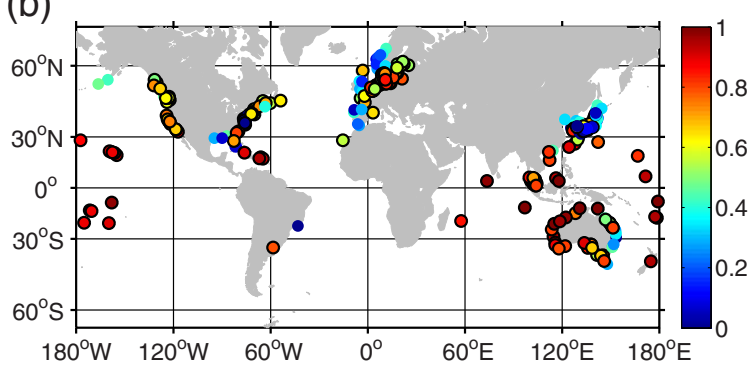

(c)

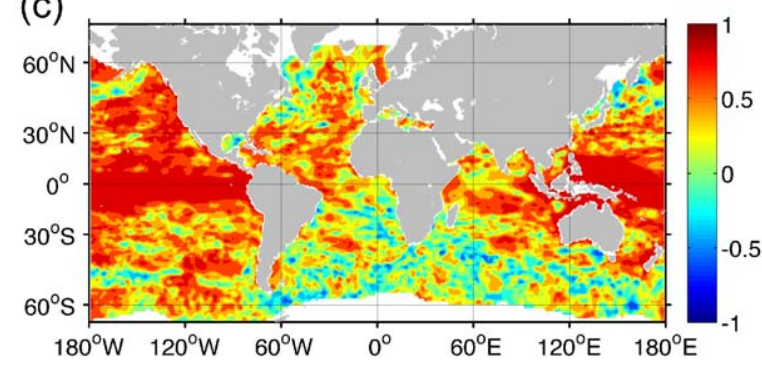

Figure 2. (a) Correlation between the sea level from SODA and that at various (236) tide gauges over the period 1960-2008. (b) Correlation between the sea level from satellite altimetry and that at various (297) tide gauges over the period 1993-2010. For the comparison with SODA (altimetry), only tide gauge records with $<20 \%(5 \%)$ of missing values over the period 1960-2008 (1993-2010) have been used. (c) Map showing the correlation at each grid point between the sea level from SODA and that from satellite altimetry over the period 1993-2008. Correlation has been computed for detrended time series of annual values. Absence of circle edge in Figures $2 \mathrm{a}$ and $2 \mathrm{~b}$ indicates non-significant correlation.
The modeled sea level fields from SODA (with all the above additional signals applied) are interpolated onto a coarser global $1^{\circ} \times 1^{\circ}$ grid (with 34,171 ocean grid points) and the seasonal cycle (computed over the period 1871-2008) is removed from each grid point by means of a harmonic analysis.

Figure 2a shows the correlation between the sea level from SODA (with the added components) and that at various tide gauges from around the world. We note that SODA performs quite well along the Western coast of the United States, in open sea islands, along the Australian coast, and in the North and Baltic Seas, with correlations above 0.7 at many stations. There are, however, various regions where its performance is significantly worse, such as along the western boundary of the Atlantic and Pacific Oceans (i.e., Eastern U.S. and Japanese coasts). Interestingly, the correlation between satellite altimetry and tide gauges shows an almost identical spatial pattern (Figure 2b). We find significant differences between tide gauges and nearby altimetry in the same regions where the model shows its worse performance. This is an indication that, in those regions, the sea level variability at the tide gauges is dominated by coastal processes that are not captured by either the model or altimetry. Note, however, that this issue is, in principle, limited to the coast and does not necessarily translate into a poor performance of the model in the deep ocean. A comparison in terms of the correlation between the model and altimetry shows that the model 


\section{QAGU Journal of Geophysical Research: Oceans}

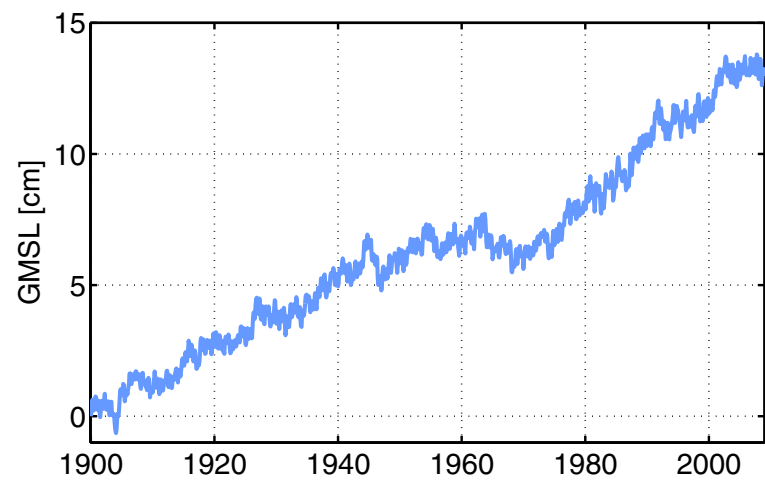

Figure 3. The GMSL from SODA after the addition of the fingerprint of continental ice mass change computed by Riva et al. [2010] and a contribution of land hydrology. does, in fact, a good job in some regions of the world oceans but a poor job in others (i.e., Southern Ocean and South Atlantic) (Figure 2c).

In order to reduce the impact of possible model adjustments to the initial conditions, we use data from 1900 onward only. The GMSL from the simulation over the period 1900-2008 is shown in Figure 3. It has a trend of $1.1 \mathrm{~mm} / \mathrm{yr}$ over the whole period and it shows significant interannual and decadal variability, in particular a drop in 1965 and an increased rate of GMSL rise from 1965 onward. This is related to an increase in the mean thermosteric component of sea level in SODA.

Overall SODA appears representative of the variability and long-term trend in GMSL reconstructions and shows regional agreement with coastal tide-gauge measurements, and so we consider it reasonable for our purposes.

\subsection{Ensemble of Sea Level Fields and Synthetic Tide Gauges}

Surrogate sea level fields are generated from SODA using an extension to multivariate data of the phaserandomized Fourier-transform algorithm [Prichard and Theiler, 1994; Christiansen et al., 2009]. Briefly, the local trend and acceleration are removed from each grid point by means of a third-order polynomial fit. Then, the Fourier transform of the SODA sea level fields is calculated at each grid point, and the phases are then randomized by rotating them at each frequency by an independent uniform random number in the range $[0-2 \pi]$, using the same random number for all grid points. This preserves all linear autocorrelations and cross correlations. In the final step, we perform an inverse Fourier transform and add the estimated third-order polynomial back to each grid point. The surrogate sea level fields generated using this algorithm have the same autocorrelations and cross correlations as the original data set [Prichard and Theiler, 1994]. Our ensemble contains 100 realizations.

Note that, in essence, the surrogate sea level fields represent the sea level variability from the original SODA fields but with different phases for all signals except the long-term trend and acceleration. The surrogate sea level fields can then be used to test the reconstruction of the fields under different tide-gauge distributions and different configurations of the RSOI method. We use synthetic tide gauges and EOFs from the surrogate sea level fields to reconstruct the GMSL. The skill of the reconstruction is then assessed by comparing the reconstructed GMSL with the average of the sea level grids over the global oceans, which is considered to be the true GMSL. The synthetic tide gauges are the model grid points where real tide gauges are available (see section 2.1 and Figure 1).

\subsection{Reconstruction Details and Statistical Parameters}

Sea level fields are reconstructed using the RSOI described by Kaplan et al. [1997, 2000]. The reconstruction methodology is briefly described in Appendix A. The first step of the RSOI method involves computing the EOFs. For the ensemble tests, EOFs are obtained from the last 20 years (1989-2008) whereas for the reconstruction with observations EOFs are calculated over the whole altimetric period (January 1993 to November 2012). We test the reconstruction for the cases with and without the EOFO (hereinafter referred to as OIH and OI, respectively). Note that a local trend is removed from each grid point prior to the computation of the EOFs, both for $\mathrm{OIH}$ and OI. The eigenvalue associated with the EOFO $\left(\lambda_{0}\right)$ is set to infinity, as in C2010. We will show in section 3 that this is the optimal setting.

The number of retained EOFs in the reconstruction is set to 10 (choosing 10 or 20 EOFs has a only a small effect on the reconstruction). We will show in section 3 that, when including the EOF0, the reconstructed GMSL does not depend on the number of retained EOFs. The observational error is set equal to $2 \mathrm{~cm}$. 
In addition to the methodology described in Appendix A, GMSL is also reconstructed as a weighted average of the tide gauges (hereinafter referred to as TG), with the weight being proportional to the cosine of the latitude where the tide gauge is located.

In order to assess the skill of the reconstruction we use three parameters: correlation between the reconstructed GMSL and the target (i.e., the true GMSL from the simulation), relative amplitude, and relative trend. Both the correlation and the relative amplitude are computed for detrended time series of annual values. Unless otherwise stated, correlations are significant at the $95 \%$ confidence level. The relative amplitude is calculated as $\left(\sigma_{\text {rec }}-\sigma_{\text {tar }}\right) / \sigma_{\text {tar }}$, where $\sigma_{\text {tar }}$ and $\sigma_{\text {rec }}$ represent the standard deviation of the target and the reconstruction, respectively. The relative trend is calculated as $\left(\tau_{\text {rec }}-\tau_{\text {tar }}\right) / \tau_{\text {tar }}$, where $\tau_{\text {tar }}$ and $\tau_{\text {rec }}$ are the trends of the target and the reconstruction, respectively. For the ensemble based on SODA, the parameters are calculated for the period 1900-1988. Thus, the calibration period (the last 20 years) is excluded. Finally, uncertainty associated with the reconstructed GMSL (i.e., the error bands in the figures) is calculated as the ensemble mean root-meansquare difference (RMSD).

\section{Theoretical Analysis of the RSOI Solution}

3.1. Derivation of GMSL Expressions for the Cases With and Without the EOFO

In this section, we rewrite equation (A5) (see Appendix A) in a notation that facilitates the interpretation of the numerical results presented later in the paper and brings out the strengths and weaknesses of the RSOI in reconstructing the GMSL. We will derive separate GMSL equations for the cases with and without the EOF0. Here we adopt the same notation as in Appendix A. Let us define the following matrices $\mathbf{U}_{i} \equiv \mathbf{H}_{i} \mathbf{E}, \mathbf{U}_{i}^{\prime} \equiv \mathbf{H}_{i} \mathbf{E}^{\prime}$. Using this notation, the RSOl solution (A5) can be written as

$$
\boldsymbol{\alpha}_{:, i}=\left(\boldsymbol{\Lambda}^{-1}+\mathbf{U}_{i}^{\top} \mathbf{R}_{i}^{-1} \mathbf{U}_{i}\right)^{-1} \mathbf{U}_{i}^{\top} \mathbf{R}_{i}^{-1} \mathbf{T}_{:, i}^{\circ}
$$

Rearranging (2) gives

$$
\boldsymbol{\alpha}_{:, i}=\left[\boldsymbol{\Lambda}^{-1}\left(I+\Lambda \mathbf{U}_{i}^{\top} \mathbf{R}_{i}^{-1} \mathbf{U}_{i}\right)\right]^{-1} \mathbf{U}_{i}^{\top} \mathbf{R}_{i}^{-1} \mathbf{T}_{:, i}^{\circ}
$$

where $\boldsymbol{I}$ is the identity matrix of order $L$. Then applying the identity $(\mathbf{A B})^{-1}=\mathbf{B}^{-1} \mathbf{A}^{-1}$ to (3) yields

$$
\boldsymbol{\alpha}_{:, i}=\left[\left(I+\Lambda \mathbf{U}_{i}^{\top} \mathbf{R}_{i}^{-1} \mathbf{U}_{i}\right)^{-1} \boldsymbol{\Lambda}\right] \mathbf{U}_{i}^{\top} \mathbf{R}_{i}^{-1} \mathbf{T}_{:, i}^{\circ}
$$

which, by applying the identity $(I+\mathbf{A B})^{-1} \mathbf{A}=\mathbf{A}(I+\mathbf{B A})^{-1}$, takes the form

$$
\boldsymbol{\alpha}_{:, i}=\boldsymbol{\Lambda} \mathbf{U}_{i}^{\top}\left(I+\mathbf{R}_{i}^{-1} \mathbf{U}_{i} \boldsymbol{\Lambda} \mathbf{U}_{i}^{\top}\right)^{-1} \mathbf{R}_{i}^{-1} \mathbf{T}_{:, i}^{\circ}
$$

Then making use of the identity $(\mathbf{A B})^{-1}=\mathbf{B}^{-1} \mathbf{A}^{-1}$, (5) can be expressed as

$$
\boldsymbol{\alpha}_{:, i}=\boldsymbol{\Lambda} \mathbf{U}_{i}^{\top}\left(\mathbf{R}_{i}+\mathbf{U}_{i} \mathbf{\Lambda} \mathbf{U}_{i}^{\top}\right)^{-1} \mathbf{T}_{:, i}^{\circ}
$$

Next, substituting equation (A4) into (6) gives

$$
\boldsymbol{\alpha}_{:, i}=\boldsymbol{\Lambda} \mathbf{U}_{i}^{\top}\left(\mathbf{R}_{\circ}+\mathbf{U}_{i}^{\prime} \boldsymbol{\Lambda}^{\prime} \mathbf{U}_{i}^{\prime \top}+\mathbf{U}_{i} \boldsymbol{\Lambda} \mathbf{U}_{i}^{\top}\right)^{-1} \mathbf{T}_{:, i}^{\circ}
$$

The first matrix $\left(\mathbf{R}_{0}=r_{0} I\right)$ inside the parentheses in (7) is a diagonal matrix containing the observational error of the tide gauges. Interestingly, the sum of the other two matrices $\mathbf{U}_{i}^{\prime} \boldsymbol{\Lambda}^{\prime} \mathbf{U}_{i}^{\prime \top}+\mathbf{U}_{i} \mathbf{\Lambda} \mathbf{U}_{i}^{\top}$ is nothing but the 
covariance matrix, $\Sigma_{i}$, of the altimetry (or model) data only at those grid points where tide-gauge observations are available at time $i$ (i.e., $\Sigma_{i}$ is an $N \times N$ matrix, where $N$ is the number of tide gauges available at time $i$ ). Note that when including the EOFO the sum $\mathbf{U}_{i}^{\prime} \boldsymbol{\Lambda}^{\prime} \mathbf{U}_{i}^{\prime \top}+\mathbf{U}_{i} \mathbf{\Lambda} \mathbf{U}_{i}^{\top}$ does not exactly coincide with the covariance matrix $\Sigma_{i}$, because it contains a contribution from the EOF0. By separating the contribution of the EOFO from that of the other EOFs, the preceding sum can be written as $\Sigma_{i}+\lambda_{0} u_{0}^{2} \mathbf{J}$, where $\lambda_{0}$ is the eigenvalue associated with the EOF0, $u_{0}$ is the value of any element of the EOFO (recall that in the EOFO all elements have the same value), and $\mathbf{J}$ is an $N \times N$ matrix of ones. This result will be used later.

Note also that when the EOFO is included in the reconstruction, the spatial-average of all other EOFs is set to zero (EOFs are later renormalized). This adjustment has a very small impact on $\Sigma_{i}$ since the variance of the sea level at tide-gauge locations is, on average, two orders of magnitude larger than that of the GMSL. As an illustration, the correlation between two GMSL reconstructions, one in which the spatial-average of all EOFs has been removed and another one in which it has not, is 0.96 for the period 1900-2011. No significant difference in the secular trends is found either.

Let us now return to equation (7). We assume now, as C2010 did, that the observational error of the tide gauges is zero (i.e., $\mathbf{R}_{\mathrm{o}}$ is a zero matrix). We will show later that this assumption does not limit the generality of the subsequent analysis. Let us also drop the temporal subscript $i$ to simplify notation. Next, we express the Ol solution $\alpha$ as a linear combination of the tide gauges contained by the column vector $\mathbf{t}^{\circ} \equiv \mathbf{T}_{:, i}^{\circ}$. We do this separately for the reconstruction with and without the EOFO.

For the case without the EOFO, (7) can be simply written as

$$
\boldsymbol{\alpha}=\Lambda \mathbf{U}^{\top} \boldsymbol{\Sigma}^{-1} \mathbf{t}^{\mathrm{o}}
$$

After some algebraic manipulation, (8) can be expressed in terms of the elements of $\Sigma^{-1}=\left(\rho_{i j}\right)$ :

$$
\alpha_{q}=\left(\lambda_{q} \sum_{i=1}^{N} u_{i q} \rho_{i 1}\right) t_{1}^{\circ}+\left(\lambda_{q} \sum_{i=1}^{N} u_{i q} \rho_{i 2}\right) t_{2}^{\circ}+\ldots+\left(\lambda_{q} \sum_{i=1}^{N} u_{i q} \rho_{i N}\right) t_{N}^{\circ} \quad q=1, \ldots, L
$$

where $\alpha_{q}$ is the temporal amplitude associated with the EOF mode $q$ (i.e., the EOF $\mathbf{U}_{:, q}$ ), $t_{i}^{0}$ is the $i$ th element of the vector of tide-gauge observations $\mathbf{t}^{\circ}, \lambda_{q}$ is the $q$-th diagonal element of $\boldsymbol{\Lambda}$, and $u_{i q}$ is the $(i, q)$ entry of the EOFs matrix $\mathbf{U}$ (i.e., the value of the EOF mode $q$ at the location of the ith tide gauge). The quantities within the parenthesis in (9) can be seen as the "weights" or "loadings" associated with each tide-gauge observation. It is also interesting to observe that the solution $\alpha_{q}$ for each EOF mode $q$ is independent of the number of retained EOFs, $L$, in the reconstruction.

Next, we deal with the case where the EOFO is included. In this case, the RSOI solution is given by

$$
\alpha=\Lambda \mathbf{U}^{\top}\left(\boldsymbol{\Sigma}+\lambda_{0} u_{0}^{2} \mathbf{J}\right)^{-1} \mathbf{t}^{\mathrm{o}}
$$

The problem here is to find a simple expression for the inverse of $\boldsymbol{\Sigma}+\lambda_{0} u_{0}^{2} \mathbf{J}$ in terms of $\boldsymbol{\Sigma}^{-1}$. By observing that the $N \times N$ matrix $\mathbf{J}$ has ones everywhere, and hence is of rank one, we can make use of the following Lemma [Miller, 1981]:

$$
(\mathbf{G}+\mathbf{H})^{-1}=\mathbf{G}^{-1}-\frac{1}{1+g} \mathbf{G}^{-1} \mathbf{H G}^{-1}
$$

where $\mathbf{G}$ is an arbitrary nonsingular square matrix, $\mathbf{H}$ is a square matrix of rank one, and $g=\operatorname{tr}\left(\mathbf{H G}^{-1}\right)$. Because the amplitude of the EOF0, $\alpha_{0}$, is the only one relevant to the GMSL, as it will be shown later, we restrict our attention to $\alpha_{0}$. By (11) and after some algebra the RSOI solution for the temporal amplitude of the EOFO (10) can be written as: 


$$
\alpha_{0}=\frac{\lambda_{0} u_{0}}{1+\lambda_{0} u_{0}^{2} \sum_{i=1}^{N} \sum_{j=1}^{N} \rho_{i j}}\left(t_{1}^{\circ} \sum_{i=1}^{N} \rho_{i 1}+t_{2}^{\circ} \sum_{i=1}^{N} \rho_{i 2}+\ldots+t_{N}^{\circ} \sum_{i=1}^{N} \rho_{i N}\right)
$$

Finally, it is convenient to write an expression for the GMSL for the cases both with and without the EOFO. In general, the GMSL is computed at each time step by

$$
\mathrm{GMSL}=\sum_{i=0}^{L} \overline{\mathbf{E}}_{:, i} \alpha_{i}
$$

where the overbar denotes average along the first dimension of the corresponding matrix (i.e., spatial average over the global oceans). When the EOF0 is not included in the reconstruction, the GMSL is calculated using the truncated EOF expansion (13), with the temporal amplitudes given by (9). However, in the case with the EOF0, we should recall that the spatial average of all EOFs except that of the EOF0 is set to zero (i.e., $\overline{\mathbf{E}}_{:, i}=0 \quad \forall i \neq 0$ ), which implies that (13) reduces to GMSL $=\overline{\mathbf{E}}_{:, 0} \alpha_{0}=u_{0} \alpha_{0}$. Now using (12), the GMSL for the case with the EOFO can be written as

$$
\mathrm{GMSL}=\frac{\lambda_{0} u_{0}^{2}}{1+\lambda_{0} u_{0}^{2} \sum_{i=1}^{N} \sum_{j=1}^{N} \rho_{i j}}\left(t_{1}^{\circ} \sum_{i=1}^{N} \rho_{i 1}+t_{2}^{\circ} \sum_{i=1}^{N} \rho_{i 2}+\ldots+t_{N}^{\circ} \sum_{i=1}^{N} \rho_{i N}\right)
$$

\subsection{Interpretation of the GMSL Equations}

The covariance matrix $\Sigma$ is always positive semidefinite thus its inverse is also positive semidefinite and hence $\sum_{i=1}^{N} \sum_{j=1}^{N} \rho_{i j} \geq 0$. This implies that the factor in front of the parentheses in (14) is a monotonically increasing function of $\lambda_{0}$, which is always positive and asymptotically approaches $\frac{1}{\sum_{i=1}^{N} \sum_{j=1}^{N} \rho_{i j}}$ as $\lambda_{0} \rightarrow \infty$. This asymptotic behavior explains why previous studies have found that, for sufficiently large values of $\lambda_{0}$ (i.e., $\lambda_{0} \mid \lambda_{0} u_{0}^{2} \sum_{i=1}^{N} \sum_{j=1}^{N} \rho_{i j} \gg 1$ ), increasing the value of $\lambda_{0}$ has almost no effect on the reconstruction of the GMSL. Since, in general, $\lambda_{0}$ is chosen so that $\lambda_{0} u_{0}^{2} \sum_{i=1}^{N} \sum_{j=1}^{N} \rho_{i j} \gg 1$, (14) can be approximated by

$$
\mathrm{GMSL}=\sum_{i=1}^{N} w_{i} t_{i}^{\mathrm{O}}
$$

where $w_{i}=\sum_{j=1}^{N} \rho_{j i} / \sum_{j=1}^{N} \sum_{k=1}^{N} \rho_{j k}$ and obviously $\sum_{i=1}^{N} w_{i}=1$.

We observe now that the weights $w_{i}$ in (15) are simply the solution of the generalized weighted mean problem [McLean et al., 2011] of the altimetry records at locations where tide gauges are available. In other words, the weights $w_{i}$ are determined so that the weighted mean $\sum_{i=1}^{N} w_{i} t_{i}^{a}$ has the smallest variance of all unbiased estimators, where $t_{i}^{a}$ represents the altimetry observations at the location of the ith tide gauge. Note that although here we refer to the values $w_{i}$ as weights, such values can in fact be negative. Expression (15) can be written in matrix form [McLean et al., 2011]:

$$
\mathrm{GMSL}=\frac{\mathbf{1}^{\top} \boldsymbol{\Sigma}^{-1} \mathbf{t}^{\mathrm{O}}}{\mathbf{1}^{\top} \boldsymbol{\Sigma}^{-1} \mathbf{1}}
$$

where $\mathbf{1}$ is a $N \times 1$ column vector of ones. It is worth noting that, if we are only interested in the GMSL, using (16) is computationally much faster and more memory-efficient than the usual algorithm (A5) since it avoids the computation of the singular value decomposition of the global altimetry data.

Note that, because the covariance matrix $\Sigma$ that appears in the expression for the generalized weighted mean (equations (15) and (16)) is derived from altimetry records rather than tide-gauge records, which can 
differ significantly (i.e., $t_{i}^{\circ} \neq t_{i}^{a}$ ), the weights $w_{i}$ in (15) minimize the variance of the weighted mean $\sum_{i=1}^{N} w_{i} t_{i}^{a}$ but not necessarily that of the weighted mean $\sum_{i=1}^{N} w_{i} t_{i}^{\circ}$. Therefore, although the variance of the generalized weighted mean is given by $1 /\left(\mathbf{1}^{\top} \boldsymbol{\Sigma}^{-1} \mathbf{1}\right)$, such expression will not yield the true variance of $\sum_{i=1}^{N} w_{i} t_{i}^{\circ}$, but only that of $\sum_{i=1}^{N} w_{i} t_{i}^{a}$. Perhaps, a more consistent approach would be to replace the inverse of the covariance matrix, $\Sigma^{-1}$, from altimetry records at tide-gauge locations by that from the tide-gauge records themselves. This would ensure that (16) is the weighted mean with minimum variance (i.e., the generalized weighted mean) for the tide-gauge records. Moreover, with this approach one could calculate $\boldsymbol{\Sigma}$ over the whole reconstruction period, which would result in a more robust weighted mean. The problem of this approach is that it is possible only if a constant tide-gauge distribution is used.

Another important feature of (16) is that it contains no information on the global covariance structure of the sea level. In other words, when the EOFO is included, the method does not benefit from the near-global coverage provided by satellite altimetry. Recall that $\Sigma^{-1}$ is calculated from altimetry data only at locations where tide gauges are available. Although, strictly speaking, the method includes some information on the correlation between the altimetry records and the GMSL when setting the spatial average of all EOFs equal to zero (this is equivalent to subtracting the GMSL from the sea level at each grid point prior to the computation of the EOFs), this correction, as commented above, results in practically the same covariance matrix $\Sigma$, and hence in the same GMSL.

Therefore, there are important differences between the case when the EOFO is used and when it is not. First, the reconstructed GMSL without the EOFO does include information on the global covariance structure provided by altimetry, as represented by the factors $u_{i q}$ in (9), but the reconstruction with the EOF0 does not. Second, the reconstructed GMSL with the EOFO is independent of the number of retained EOFs, $L$, whereas that without the EOFO is not.

When including the EOF0, the reconstruction of the GMSL is simply a weighted mean of tide gauges based on information from altimetry records only at tide-gauge locations. Recalling that $\Sigma^{-1}$ represents the inverse of the covariance matrix of altimetry records at tide-gauge locations, its elements, $\rho_{i j}$, have a convenient interpretation in terms of linear regression. The diagonal elements $\rho_{j j}$ can be expressed as $\rho_{j j}=\frac{1}{\left(1-R^{2}\right) \sigma_{i}^{2}}$, where $R^{2}$ is the coefficient of determination (i.e., the explained variance) obtained from regressing the altimetry record at the location of the $j$-th tide gauge on the altimetry records at all other tidegauge locations, and $\sigma_{j}^{2}$ is the variance of the altimetry record at the jth tide-gauge location. The offdiagonal elements, $\rho_{i j}$, are related to the coefficients, $\beta_{i j}$, in the aforementioned regression by $\rho_{i j}=-\beta_{i j} \rho_{j j}$, where $\beta_{i j}$ is the regression coefficient associated with the altimetry record at the ith tide-gauge location. Hence the factors determining the contribution of each tide-gauge record to the GMSL in (15) can be written as

$$
\sum_{i=1}^{N} \rho_{i j}=\frac{1}{\left(1-R^{2}\right) \sigma_{j}^{2}}\left(1-\sum_{i \neq j} \beta_{i j}\right)
$$

From (17), we observe that, in general, tide-gauge locations where altimetry records show high correlation with other altimetry records, either from nearby or distant grid points, will result in higher values of $R^{2}$ and hence in larger weights, especially where records have relatively small variance. Note that as $R^{2}$ nears 1 the factor $\frac{1}{\left(1-R^{2}\right)}$ increases quickly. Nearby tide-gauge records are in general highly correlated with each other. Hence regions with a high density of stations and with reasonably good agreement with altimetry will be given a large weight in the reconstruction whether or not they are representative of the open-ocean sea level variability. This may result in a poor reconstruction of the GMSL variability and the trend if a specific area is subject to regional forcing not linked with GMSL and/or if, for example, GIA models have significant errors in these areas. In addition, because (16) is a weighted mean of tide gauges, the reconstructed GMSL with the EOFO will, in general, overestimate the variability when compared with the reconstruction without the EOFO because tide-gauge records tend to show relatively large variability.

When the EOF0 is not used in the reconstruction the factors $u_{i q}$ in (9) include information on the variance explained by the global EOF mode $q$ at the ith tide gauge and the aforementioned problems are removed. 
For instance, we know that a significant fraction of the interannual GMSL variability from altimetry is associated with the ENSO, which is represented by the EOF1. When the EOFO is not included, (9) uses this global information contained in the EOF1 by giving less weight to locations that are not correlated with the ENSO, which results in a better reconstruction of the GMSL variability linked to the ENSO.

An issue that affects the reconstruction both with and without the EOFO is the fact that very small (near zero) eigenvalues of the covariance matrix $\Sigma$ have a very large contribution to $\Sigma^{-1}$. This can be seen by rewriting (17) in terms of the eigenvalues, $s_{k}$, and eigenvectors, $\mathbf{D}=\left(d_{i j}\right)$, of $\Sigma$ :

$$
\sum_{i=1}^{N} \rho_{i j}=\sum_{i=1}^{N} \sum_{k=1}^{N} \frac{d_{j k} d_{i k}}{s_{k}}
$$

From (18), it is obvious that near zero eigenvalues will have the largest influence. Now note that because the smallest eigenvalues are those with larger uncertainty in their estimate, their influence on $\Sigma^{-1}$ can significantly degrade the performance of the GMSL reconstruction. Recall that, in general, the EOFs associated with the lowest eigenvalues represent small-scale variability, or in the case where there is nonuniform distribution of tide gauges may also represent regional-scale variability. Naturally, we do not want $\Sigma^{-1}$, and thus the estimate of the weights, to be dominated by the noisy smallest-scale structures but we would want tide gauges which are the sole information in large parts of the ocean to be given appropriate weight. One remedy to this problem is to truncate the small eigenvalues in the calculation of $\Sigma$ and then diagonally load the estimated covariance matrix to ensure that $\boldsymbol{\Sigma}$ is invertible. However, this is not necessary if we realize that a similar effect is achieved when the observational error contained by the diagonal elements of $\mathbf{R}_{\mathrm{o}}$ is set to a nonzero value. This is in essence equivalent to diagonal loading of the covariance matrix and has the effect of greatly reducing the influence of those near zero eigenvalues over inversion. Setting the diagonal elements of $\mathbf{R}_{\mathrm{o}}$ to a nonzero value can also be interpreted as a type of regularization, a ridge-like regularization to be more precise [Hoerl and Kennard, 1970], since its effect is that of adding a constant to the diagonal of the covariance matrix $\boldsymbol{\Sigma}$. Therefore, all conclusions and equations presented in this section are still valid when $\mathbf{R}_{0} \neq 0$ as long as one replaces $\rho_{i j}=\sum_{k=1}^{N} \frac{d_{i k} d_{j k}}{s_{k}}$ by $\rho_{i j}=\sum_{k=1}^{N} \frac{d_{i k} d_{j k}}{s_{k}+r_{0}}$. Note that, if $\mathbf{R}_{0} \neq 0$, the weights $w_{i}$ in (15) are the solution of the generalized weighted mean problem with regularization, in which the level of regularization depends on the value of $r_{0}$.

\section{Numerical Experiments}

Here we present the results of various numerical experiments aimed at verifying some of the conclusions derived from the analysis of the RSOI solution as well as assessing the skill of the RSOI in reconstructing the

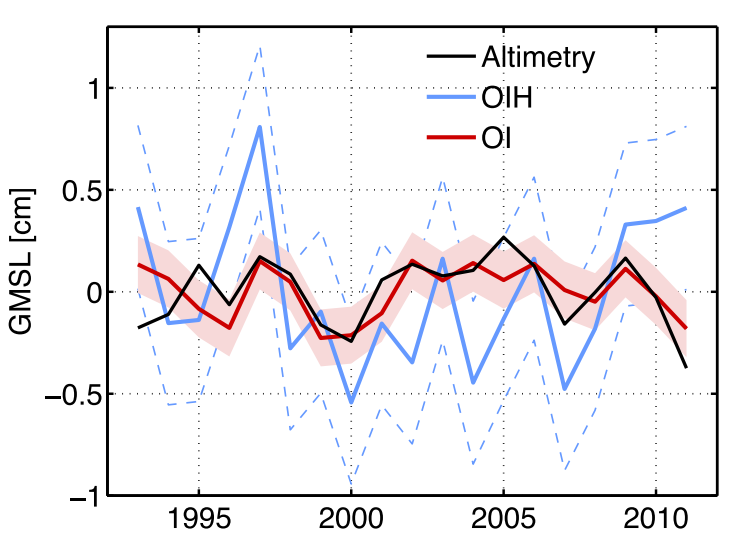

Figure 4. Comparison of the GMSL from satellite altimetry (black) with the reconstructed GMSL for both OIH (blue) and OI (red). All time series have been detrended. Shading and dashed lines represent uncertainty $( \pm 1 \sigma)$, which has been calculated as the RMSD between the target and the reconstruction.
GMSL. Before proceeding to the results based on the ensemble of sea level fields described in section 2.5, it is convenient to first look at a comparison of the GMSL from altimetry with the estimates provided by $\mathrm{Ol}$ and $\mathrm{OIH}$ (using real tide gauge and altimetry observations) after the trend for 1993 to 2011 is removed (Figure 4). A noticeable feature is that $\mathrm{OIH}$ shows no correlation with altimetry and overestimates the GMSL variability with a relative amplitude of 1.2, in spite of the fact that the comparison is for the calibration period. Although strictly speaking the OIH solution is not statistically incorrect since the curve from altimetry is mostly within the uncertainty band associated with $\mathrm{OlH}$, it is important to note that, as we showed in section 3, part of the difference between the two curves is due to deficiencies in the methodology when including 


\section{(a)}

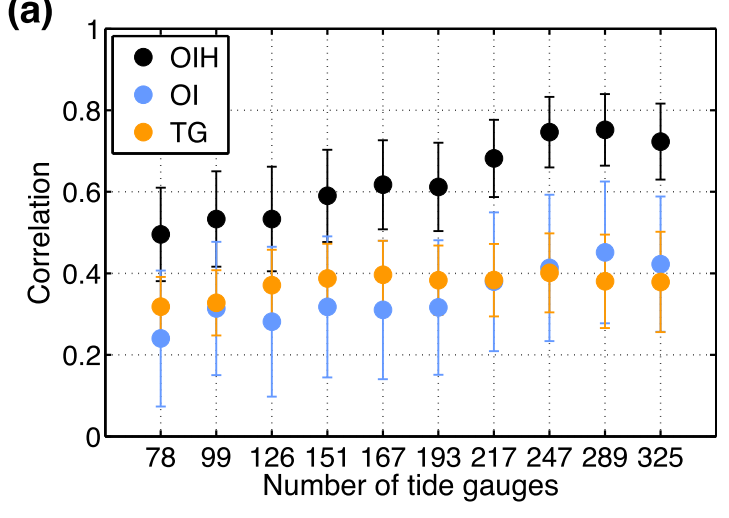

(b)

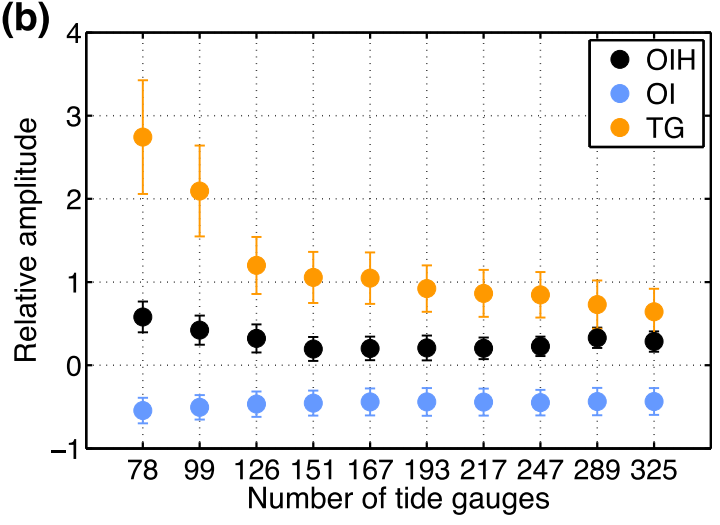

(c)

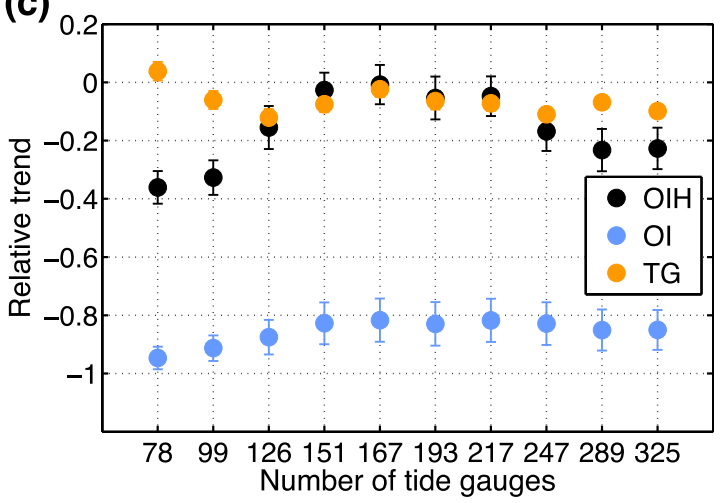

Figure 5. (a) Correlation, (b) relative amplitude, and (c) relative trend for different tide-gauge distributions, and for OIH (black), OI (blue), and TG (orange). Results are from the ensemble of surrogate sea-level fields based on SODA. The dots represent the ensemble mean while the error bars denote one standard deviation. the EOFO. Conversely, OI captures both the variability observed in altimetry and its magnitude with a significant correlation of 0.61 and a relative amplitude of -0.2 .

We proceed now to the results of the numerical experiments based on the ensemble of sea level fields. We begin by testing the reconstruction under different tide-gauge distributions. In particular, we select 10 different tide-gauge distributions from the set of composite records described in section 2.1. In this first experiment, the tide-gauge distribution does not change over time (i.e., the number and location of tide gauges is the same for each month). Note, however, that in practice the GMSL is usually reconstructed using a time-varying tide-gauge distribution, in which both the location and the number of tide gauges change over time. It is, therefore, important to assess whether this has an effect on the skill of the reconstruction. To do this, we will conduct a second experiment using a time-varying tide gauge based on the actual distribution for each month shown in Figure 1.

Figure 5 shows the ensemble mean correlation, relative amplitude and relative trend for $\mathrm{OIH}, \mathrm{Ol}$, and TG and for each of the 10 constant tide-gauge distributions. In terms of the correlation (Figure 5a), OlH performs better than both OI and TG for all tide-gauge distributions, with ensemble mean correlations ranging from 0.50 to 0.75 . Ol and TG show similar correlations with ensemble mean values ranging from 0.25 to 0.45 . Note that these correlations are statistically significant. The ensemble mean relative amplitude ranges from 0.2 to 0.6 for $\mathrm{OlH},-0.6$ to -0.4 for Ol, and 0.7 to 2.8 for TG (Figure $5 \mathrm{~b}$ ). Looking at the relative trend (Figure $5 \mathrm{c}$ ) shows that OI underestimates the GMSL trend significantly, with an ensemble mean worse than $-80 \%$ for all tide-gauge distributions. However, OlH capture the trend much better, with an ensemble mean relative trend rang-

ing from -23 to $0 \%$ for the last eight distributions. The underestimation is larger ( $-35 \%)$ for the first two distributions and it appears increasing again for the two largest distributions. Finally, TG appears to perform slightly better than $\mathrm{OIH}$ with an ensemble mean relative trend ranging from -11 to $4 \%$ for all distributions. Note that the relatively strong underestimation of the trend in OlH for the first two distributions simply reflects the fact that the influence that each tide gauge has on the reconstructed trend is determined on the basis of a covariance matrix derived from deseasonalized monthly time series. It turns out that for these two distributions, tide gauges with smaller trends are given the largest weights, which results in an underestimation of the trend. 
Overall, these results are consistent with those of C2010, who also found that OlH outperformed both OI and TG in terms of the reconstruction of the interannual variability. The overestimation of the variability (as given by the relative amplitude) by $\mathrm{OIH}$ is somehow larger in the study of C2010. This is in part due to the fact that, unlike us, C2010 set the value of the observational error $\left(r_{0}\right)$ equal to zero, which, we have found, results in a larger variability of the reconstructed GMSL. They also found that all methods underestimated the long-term trend, and neither OIH nor Ol were better than TG. Nevertheless, it is important to recall that when observations are used instead of synthetic tide gauges, Ol outperforms OlH in terms of capturing the interannual variability (Figure 4).

The main reason for this inconsistency between the results based on models here and in C2010 and reconstructions where observations are used is that, in the model, coastal and deep-ocean sea level are more highly correlated than in the real world, which results in a higher correlation between synthetic tide gauges and GMSL. Note from (15) and (17) that records with relative small variances and that are correlated with other records are given positive large weights regardless of whether they show negative or no correlation with the GMSL. This, of course, results in a poor reconstruction of the GMSL variability. As discussed in section 3, this is not necessarily the case when the EOFO is not used as long as the processes causing the negative correlation between the tide gauges and the GMSL are represented by the retained EOFs.

It is also interesting to note that because the largest weights in (15) are usually positive, the reconstructed GMSL when using the EOFO will be, in general, correlated with the average of the tide gauges, at least much more so than when not using the EOF0. As an example of this, we have found that for the constant distribution with 289 tide gauges, the ensemble mean correlation between the OIH and TG is 0.72 , whereas it is only 0.38 between OI and TG.

In an attempt to reduce the differences between synthetic and real tide gauges, we perturb the synthetic tide gauges in SODA so that they have exactly the same correlation with the GMSL as real tide gauges. This is done as follows. Let $\mathbf{y}_{i}$ and $\boldsymbol{\eta}$ be the ith synthetic tide-gauge record and the GMSL from SODA (both detrended), respectively. Then the corresponding perturbed synthetic tide gauge, $\hat{\mathbf{y}}_{i}$, is obtained as

$$
\hat{\mathbf{y}}_{i}=\sigma_{i}\left(\rho_{\text {real }} \frac{\boldsymbol{\eta}}{\sigma_{\eta}}+r_{i} \sqrt{1-\rho_{\text {real }}^{2}}\right)
$$

where $\sigma_{i}$ and $\sigma_{\eta}$ are, respectively, the standard deviations of $\mathbf{y}_{i}$ and $\boldsymbol{\eta}, \rho_{\text {real }}$ is the correlation between the corresponding ith real tide-gauge record and the GMSL from altimetry, and $r_{i}$ is the residual from the simple linear regression of $\frac{\mathbf{y}_{i}}{\sigma_{i}}$ on $\frac{\eta}{\sigma_{\eta}}$. Similar approaches have been used in pseudoproxy experiments of climate reconstructions where pseudoproxies are perturbed to mimic the characteristics of real proxies [Christiansen

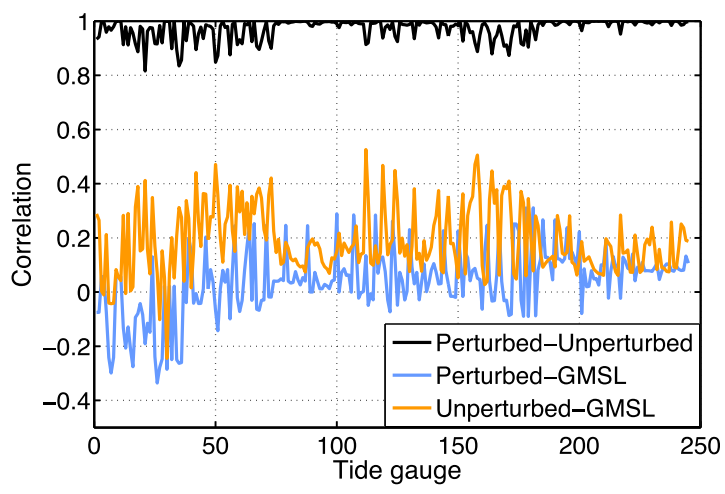

Figure 6. Correlation between perturbed and unperturbed synthetic tide gauges (black), and between unperturbed (orange)/perturbed (blue) synthetic tide gauges and the GMSL from SODA over the period 1900-2008. Correlation has been computed for detrended time series of annual values. Perturbed synthetic tide gauges have been obtained using equation (19). and Ljungqvist, 2012; Smerdon, 2012]. Transformation (19) ensures that $\hat{\mathbf{y}}_{i}$ and $\boldsymbol{\eta}$ have correlation $\rho_{\text {real }}$, and that the part of $\hat{\mathbf{y}}_{i}$ that is not correlated with $\boldsymbol{\eta}$ is still related to the unperturbed tide-gauge record $\mathbf{y}_{i}$. It also ensures that $\mathbf{y}_{i}$ and $\hat{\mathbf{y}}_{i}$ have nearly the same variance. Once $\hat{\mathbf{y}}_{i}$ has been calculated we add back the long-term trend so that it has the same trend as the unperturbed synthetic tide gauge. As an illustration of the effect of this modification, we have compared the correlation of the GMSL from SODA with both unperturbed and perturbed tide gauges (Figure 6). As commented above, unperturbed tide gauges show, in general, much higher positive and less negative correlations with the GMSL than perturbed (and real) tide gauges. Note however that (19) changes the variability of the synthetic tide gauges only 

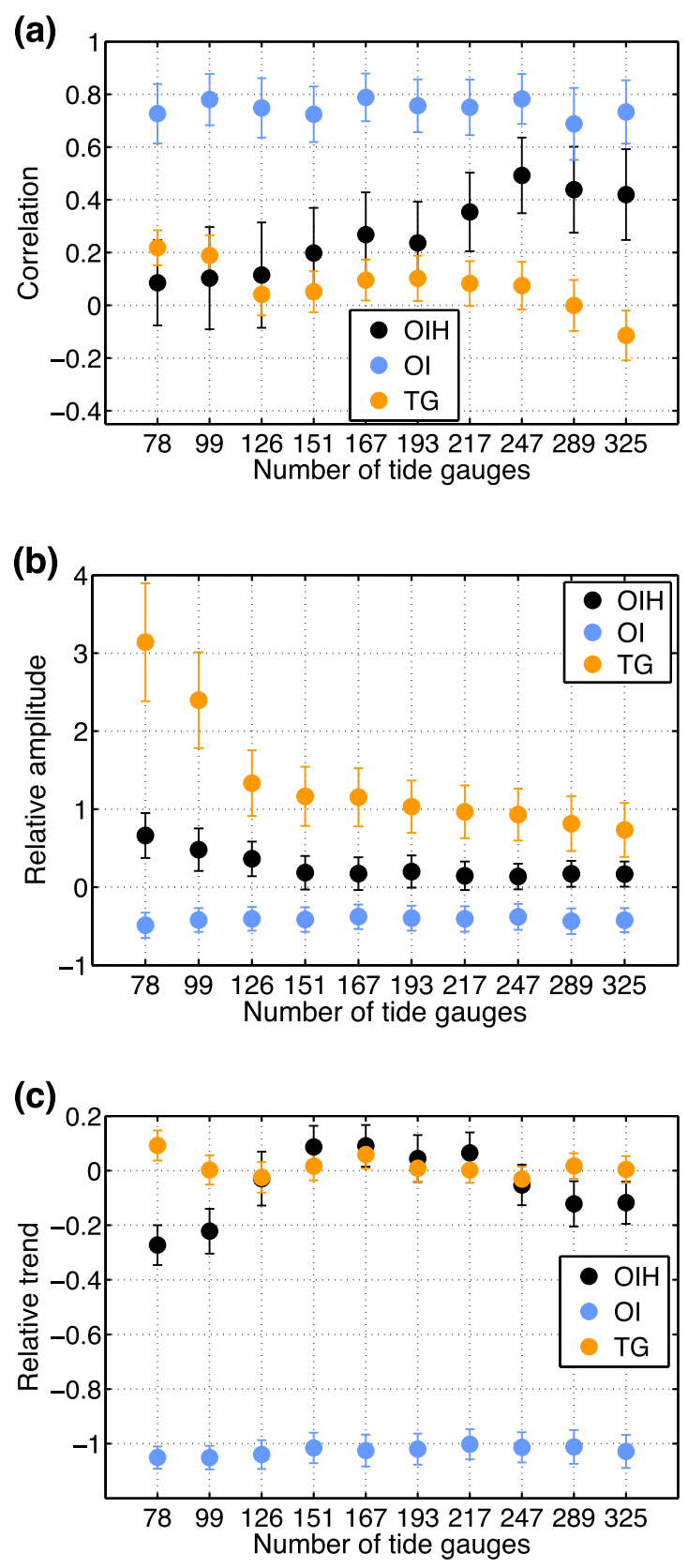

Figure 7. The same as Figure 5 but for perturbed synthetic tide gauges. slightly, as exemplified by the high correlation $(>0.8)$ between the unperturbed and perturbed tide gauges (Figure 6).

Next, we repeat the experiments for the 10 constant tide-gauge distributions but using now the perturbed tide gauges. Note that this approach assumes that the processes driving the sea level variability at the timescales resolved by altimetry are similar to those at longer timescales (i.e., multidecadal). Results of the experiments are shown in Figure 7. We observe that, after perturbing the tide gauges, OI performs quite well in capturing the GMSL variability with an ensemble mean correlation of $\sim 0.7$ for all distributions. Conversely, OlH now shows much smaller correlations than for the case with unperturbed tide gauge. Regarding the relative amplitude, $\mathrm{OIH}$ shows values ranging from 0.1 to 0.7 , whereas Ol shows values of about -0.4 for all distributions. We can conclude, therefore, that errors in the Ol estimate are primarily due to a difference in the amplitude of the variations (i.e., Ol captures the variability reasonably well but underestimates its magnitude), whereas those in the OlH estimates are due to a difference in both the amplitude and the phase of the variations. Finally, results for the reconstruction of the long-term trend are similar to those with unperturbed tide gauges. OlH gives a reasonable estimate of the trend with an ensemble mean trend difference ranging from $-25 \%$ to $10 \%$ for all distributions whereas OI completely underestimates it for all distributions. It is worth noting that OIH does not always underestimate the trend but, in some cases, it can also overestimate it.

Let us now explore the reconstruction when using the time-varying tide-gauge distribution with perturbed tide-gauge records. We begin by showing a comparison between the target and the OlH estimate for one of the ensemble realizations (Figure 8). For this particular realization, the correlation between the target and the reconstructed GMSL is 0.3 , the relative ampli-

tude is 0.3 , and the relative trend is $-11 \%$. We note that, although both curves exhibit significant decadal and multidecadal variability, such variability is not well captured by the reconstruction. For instance, there are various periods (e.g., 1900-1920 and 1940-1960) in which the target is outside the error band associated with the $\mathrm{OIH}$ curve, reflecting the fact that its decadal and multidecadal variability differs significantly from that of $\mathrm{OIH}$. It is also interesting to note that the variability shown by $\mathrm{OIH}$, especially prior to 1960 , is significantly larger than that shown by the target, confirming that $\mathrm{OlH}$ tend to overestimate the variability.

Ensemble averages for the case of a time-varying distribution show that, in terms of the correlation, Ol gives the best result with an ensemble mean value of $0.71 \pm 0.13$ as compared to a value of $0.33 \pm 0.15$ for $\mathrm{OlH}$. The ensemble mean relative amplitude is $-0.45 \pm 0.11$ for Ol and $0.29 \pm 0.15$ for $\mathrm{OlH}$. With regards to the 


\section{QAGU Journal of Geophysical Research: Oceans}

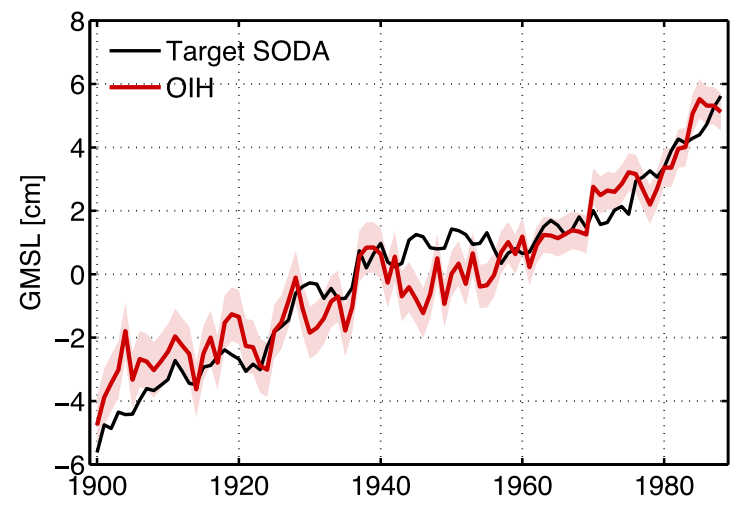

Figure 8. A comparison between the GMSL from SODA (black) and OIH (blue) for a time-varying tide-gauge distribution (based on one of the ensemble realizations). Shading represents uncertainty $( \pm 1 \sigma)$. trend, OIH shows a underestimation of the trend of $-12 \%$ whereas Ol completely underestimate it. Note that the correlation for $\mathrm{OlH}$ is rather at the lower end of the range of the 10 constant distributions, which indicates that, when including the EOF0, using a time-varying tide-gauge distribution slightly degrades the skill of the reconstruction.

\section{GMSL Reconstruction for the Period 1900-2011}

Here the GMSL is reconstructed both with and without the EOFO and using the whole set of real tide gauges described in section 2.1 (i.e., a time-varying tide-gauge distribution) for the period 1900-2011. EOFs are computed from

satellite altimetry over the whole period (January 1993 to November 2012). We use 10 EOFs and a value of $2 \mathrm{~cm}$ for the observational error. In order to avoid issues with different datum levels in the real tide-gauge data, and following Church et al. [2004], here we use sea level differences.

In section 3, we have shown that, in general, only OI is able to capture the GMSL interannual variability from altimetry (see also Figure 4). It is well known that interannual variations in GMSL are significantly correlated with the ENSO index [Nerem et al., 2010]. The signals associated with the ENSO are primarily represented in the altimetry data by the first EOF, which explains why Ol is able to capture such signals. In essence, tidegauge locations that are more affected by the ENSO are given larger weights in the estimate of $\alpha_{1}$ through the factors $u_{i q}$ in (9). Note that other climate signals may also be captured by Ol as long as they are represented by the retained EOFs. The fact that (16) does not represent spatially weighted averages and does not identify which tide gauges are more influenced by climate signals like the ENSO explains why OIH does not capture such signals.

We can, therefore, use time series of the ENSO index as a proxy for the interannual GMSL variability to validate the reconstruction for the period not covered by altimetry, similar to Chambers et al. [2002]. Note, however, that this approach assumes that the relationship between GMSL and the ENSO remain unchanged before the altimetry period. As evidence of the relationship between GMSL and the ENSO index, we have found correlations of 0.65 (annual values) and 0.40 (monthly values) between detrended time series from altimetry and the ENSO index for the period 1993-2011 (both significant at the 95\% confidence level). Similarly, we can also use time series of land hydrology changes as provided by (1) using the CPC global monthly soil moisture data set as they have been shown to be significantly correlated with the GMSL form altimetry [Cazenave et al., 2012]. Changes in land hydrology are closely linked to the ENSO and they provide an estimate not only of the sea level variability but also of its magnitude. As an example of their relationship with the GMSL, we find that land hydrology changes account for $\sim 40 \%$ of the variance in the GMSL from altimetry for annual values.

A comparison between our Ol estimate and the ENSO index for the period 1900-2011 is shown in Figure 9a. Features associated with long timescales have been removed by applying a high-pass filter with a cutoff period of 10 years to both time series. The Ol estimate and the ENSO index agree very well with a correlation of 0.80 ( 0.60 for unfiltered annual values) over the period $1900-2011$. We also note that the agreement between the two curves does not degrade significantly as we move backward in time and, hence, as the number of tide gauges decreases. In particular, the correlations for the first (1900-1955) and last (19562011) 56 years are 0.71 and 0.87 , respectively. A similar result was obtained by Chambers et al. [2002] for the period 1950-2001. Moreover, they verified the relationship in the 1980s during the large La Niña event of 1987 using independent Geosat altimeter measurements. There is no significant correlation between OlH and the ENSO index.

Ol is also in good agreement with changes in land hydrology over the period 1948-2011 on all timescales (Figure 9b), with correlations of 0.72 and 0.87 for annual and low-pass filtered (5 year cutoff) values, 
(a)
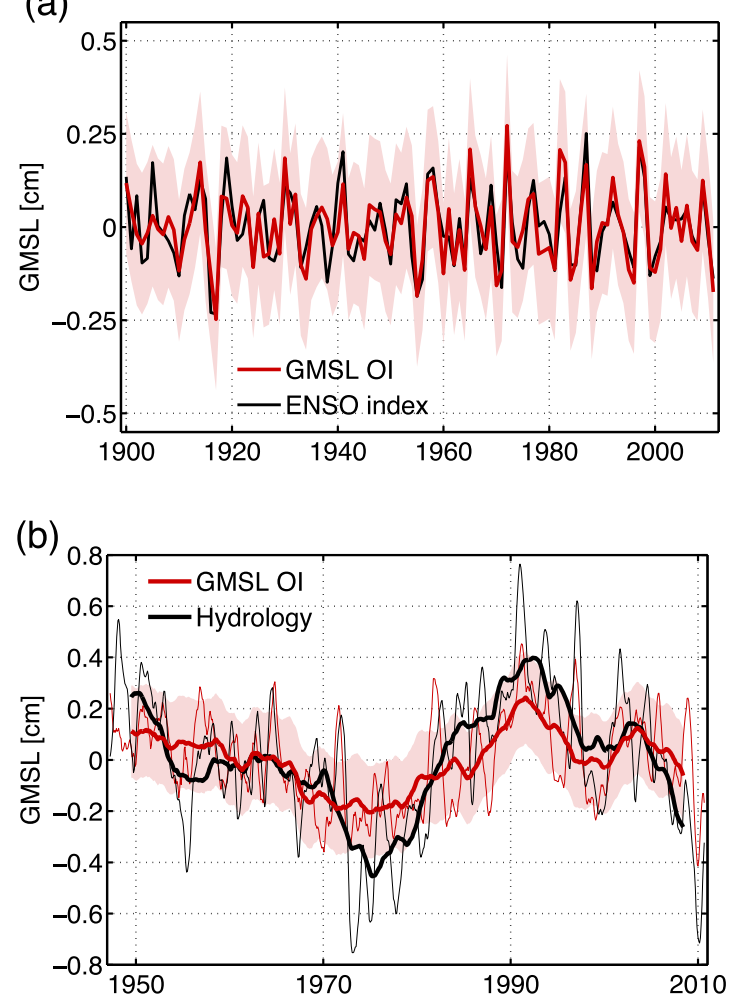

(c)

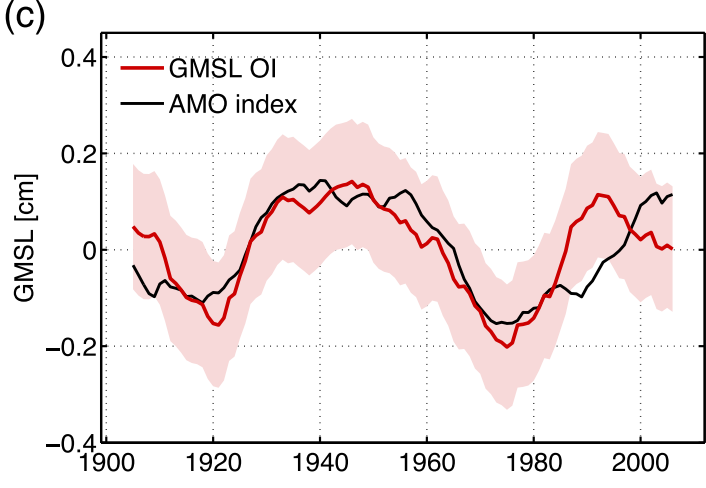

Figure 9. (a) Comparison of the 10 year high-pass filtered OI GMSL (red) with the ENSO index (black). (b) Comparison of the 5 year low-pass filtered OI GMSL (red) with land hydrology changes (black). The 6 month low-pass filtered time series (thin lines) are also shown. (c) Comparison of the 10 year low-pass filtered OI GMSL (red) with the AMO index (black). Shading represents uncertainty $( \pm 1 \sigma)$. All time series have been detrended. respectively. Changes, however, are 40\% smaller in Ol, suggesting an underestimation. The good agreement between land hydrology changes and altimetry suggests that these multidecadal fluctuations in OI may very well be a true GMSL signal of hydrological origin. Note also that the magnitude of the variability in our low-pass filtered Ol estimate (peak-topeak fluctuations of $\sim 2 \mathrm{~mm}$ ) is similar to that in the reconstructed GMSL of Chambers et al. [2002]. Finally, as expected, there is no significant correlation between $\mathrm{OIH}$ and land hydrology changes.

To further investigate the decadal and multidecadal variations shown by $\mathrm{Ol}$ and also land hydrology changes we have conducted some comparison with various climate indices. We have first compared the low-pass filtered (10 year moving average and detrended) time series of the reconstructed GMSL with the ENSO index, but we have found no significant correlation over the period 1900-2011, neither for OIH nor for Ol. We have found, however, a significant correlation of 0.78 between $\mathrm{OI}$ and the AMO index (Figure 9c). Both Ol and the AMO index show a quasi 60 year oscillation ( $\sim 2 \mathrm{~mm}$ peak-to-peak) with minimum values around 1920 and 1980, consistent with the results of Chambers et al. [2012]. Note that, although the 60 year oscillations in Ol and in the curves of Chambers et al. [2012] coincide in phase, the magnitude of the oscillations in the latter is an order of magnitude larger. Surprisingly, OlH shows a nonsignificant negative correlation of -0.44 with the $\mathrm{AMO}$ index.

Finally, we have compared our OlH estimate with the GMSL estimates of CW2011 and Jevrejeva et al. [2008] over the period 1900-2011 (not shown). The long-term trends over the common period 1900-2002 are $1.9 \pm 0.2 \mathrm{~mm} /$ $\mathrm{yr}, 1.6 \pm 0.2 \mathrm{~mm} / \mathrm{yr}$, and $1.9 \pm 0.2 \mathrm{~mm} / \mathrm{yr}$ for our OIH estimate, CW2011, and Jevrejeva et al. [2008], respectively. Our OlH estimate of the

GMSL trend is in good agreement with that of Jevrejeva et al. [2008] and larger than that of CW2011. After the removal of a linear trend, our OIH estimate is very similar to those of CW2011 and Jevrejeva et al. [2008], with correlations of 0.76 and 0.69 , respectively. Finally, it is worth commenting that decadal fluctuations in the OlH estimate and also in the reconstructions of CW2011 and Jevrejeva et al. [2008] are $\sim 5$ times greater than those in both our Ol estimate and the reconstructed GMSL of Chambers et al. [2002].

\section{Conclusions and Discussion}

In this study, we have explored the skill of the RSOI method in reconstructing the GMSL. Our goal has been to assess how well the GMSL variability can be reconstructed and to explore the sensitivity of the method 
to different configurations, including the use of the EOFO. The EOFO was first introduced by Church et al. [2004] in order to account for uniform sea level changes. While adding the EOF0 certainly improves the reconstruction of the long-term trend, it alters the reconstruction of the interannual and decadal variability significantly.

In section 3, we have presented a theoretical analysis of the RSOI solution for the cases both with and without the EOF0. We have found that when the EOFO is used the GMSL is essentially a weighted mean of the available tide gauges with the weights being the solution of the generalized weighted mean problem (with regularization when $r_{0} \neq 0$ ) of the altimetry records at the tide gauge locations, and thus the GMSL contains no information on the global covariance structure of sea level. The weights in the weighted mean are determined based on the relative variances and correlations among tide gauges at interannual timescales. In particular, tide-gauge records that have relatively small variance and significant correlation with other tide gauges are given the largest weights whether or not they are representative of the open-ocean sea level (see equations (15) and (17)). As a result, when the EOFO is used, the reconstructed GMSL is unable to capture climate signals like those associated with the ENSO, which tend to be represented by the leading global EOFs. Our analysis indicates that the decadal and multidecadal variability in the GMSL reconstructions with the EOF0 reflects regional coastal variability as seen by the tide gauges rather than a true global variation. In other words, the reconstructed GMSL does not properly account for the internal climate variability. When the EOFO is not used, the reconstructed GMSL uses information on the global covariance structure provided by altimetry, as represented by the factors $u_{i q}$ in (9), and thus climate signals such as those associated with the ENSO are, in principle, well captured by the reconstruction method.

It is also important to observe that, although the long-term trend seems to be reasonably well captured when using the EOF0, using the covariance matrix of deseasonalized monthly time series as the basis for determining the contribution of each tide gauge to the trend is dubious because it assumes that the interannual variability and the trend are driven by the same mechanisms. Nevertheless, it is worth commenting that the biases in trend estimates with the EOFO are usually no larger than $25 \%$ in absolute value.

The numerical experiments performed in section 4 have confirmed the conclusions from the theoretical analysis of the RSOI solution. Comparing the GMSL from altimetry with the reconstructed GMSL shows that not including the EOFO results in a better reconstruction of the interannual variability. Including the EOF0 leads to a GMSL reconstruction that overestimates the interannual variability and has no significant correlation with the GMSL from altimetry. The results from the ensemble of sea level fields based on SODA have confirmed these results. When using synthetic tide gauges from SODA, results suggest that both the variability and the long-term trend are better reconstructed with the EOFO, but this is only because synthetic tide gauges in the model are more representative of the open-ocean sea level variability than real tide gauges. After perturbing the synthetic tide gauges so that their variability is more consistent with that of real tide gauges, results show that not including the EOFO leads to a better reconstruction of the variability. It is also worth commenting that, while the long-term trend is much better reconstructed when using the EOF0, even in this case a simple average of tide gauges seems to provide a better estimate of the trend. Finally, we have also found that using a time-varying tide-gauge distribution slightly degrades the skill of the reconstruction.

In this study, we have also presented a GMSL reconstruction for the period 1900-2011. We have used long time series of the ENSO index as a proxy for GMSL interannual variability, similar to Chambers et al. [2002]. Our estimate of the GMSL without the EOF0 shows, at interannual timescales, a significant correlation (0.80) with the ENSO index for the period 1900-2011. Conversely, no significant correlation with the ENSO index is found when the EOFO is included. This confirms that climate signals like the ENSO, whose effect on the GMSL from altimetry is so remarkable [Nerem et al., 2010; Llovel et al., 2011], are only captured when the EOFO is not included.

The skill of the reconstruction on decadal or longer timescales has been assessed by comparing the GMSL with time series of land hydrology changes, which we know explain a significant fraction of the GMSL from altimetry. We have found a good agreement between the reconstruction without the EOF0 and land hydrology changes over the period 1948-2011 at all timescales, including decadal and multidecadal. We note, however, that OI may underestimate the amplitude of the variations by about $40 \%$. No correlation has been found for the reconstruction with the EOFO. We have also found a significant correlation (0.78) between the low-pass filtered GMSL without the EOFO and the AMO index for the period 1900-2011. Both the 
reconstructed GMSL and the AMO index show a quasi 60 year oscillation with minimum values around 1920 and 1980, consistent with the results of Chambers et al. [2012]. Interestingly, the GMSL with the EOF0 also shows a quasi 60 year oscillation (not shown), however, the maximum and minimum of such oscillation do not coincide with those of the AMO index and their magnitude is $\sim 5$ times greater. We have also compared our GMSL with the EOF0 with the estimates of CW2011 and Jevrejeva et al. [2008]. In terms of their interannual and interdecadal variability, all estimates agree very well with correlations above 0.70 .

Our results raise doubts about the reliability of the interannual to multidecadal variability in the GMSL reconstructions that use the EOF0. The theoretical and numerical results presented here suggest that the multidecadal variability in such reconstructions reflects regional variability (primarily associated with changes in atmospheric forcing) as seen by the tide gauges, rather than a true global signal. Such variability can have a significant effect on estimates of acceleration in the rate of GMSL rise since it can largely mask a possible underlying mean acceleration due to global warming or can result in the detection of spurious accelerations. This should be considered when attempting to interpret accelerations in the rate of GMSL rise derived from sea level reconstructions.

The deficiencies of the tide-gauge network in terms of spatial nonuniformity and the changing in time numbers of available tide gauges cannot be overcome by the statistical reconstruction of GMSL and the limitations of such estimates should be kept on mind when statements about global sea level trend and acceleration are made.

\section{Appendix A}

In this appendix, we briefly describe the details of the reconstruction methodology. Basically, the first step of the method involves calculation of the EOFs from a spatially dense data set (e.g., satellite altimetry). Then, for each time step (months) the temporal amplitudes of a truncated set of EOFs (the reduced space) are estimated by minimizing a cost function that fits, in a weighted least square sense, a linear model to a set of long but spatially sparse observations (i.e., tide gauges).

In the following, we describe the reconstruction methodology, using the same notation as in Kaplan et al. [2000] and C2010. Let the $n \times m$ matrix X denote a set of spatially dense sea level observations (e.g., from altimetry) with $n$ variables (i.e., grid points) and $m$ temporal samples. The longer but spatially sparser set of tide-gauge observations is arranged in an $N \times M$ matrix $\mathbf{T}^{\circ}$ that consists of $M$ temporal samples at $N$ different locations.

We begin by calculating the covariance matrix of the data matrix $\mathbf{X}$, represented by the $n \times n$ matrix $\mathbf{C}$, in its canonical form:

$$
\mathbf{C}=\mathbf{E} \Lambda \mathbf{E}^{\top}+\mathbf{E}^{\prime} \Lambda^{\prime} \mathbf{E}^{\prime \top}
$$

where $\boldsymbol{\Lambda}$ is a diagonal matrix of order $L$ that contains the $L$ largest eigenvalues, and $\Lambda^{\prime}$ is diagonal matrix of order $(n-L)$ containing the $(n-L)$ discarded eigenvalues. In addition, $\mathbf{E}$ and $\mathbf{E}^{\prime}$ are $n \times L$ and $n \times(n-L)$ matrices whose columns are the EOFs corresponding to the eigenvalues contained in $\boldsymbol{\Lambda}$ and $\Lambda^{\prime}$, respectively. The superscript $^{\top}$ denotes matrix transpose. Then, the sea level field $\mathbf{T}(n \times M)$ can be approximated by a sum of the contributions of the retained EOFs modes $\mathbf{E}$, each weighted by the corresponding temporal amplitude $\alpha(L \times M)$,

$$
\mathbf{T}=\mathbf{E} \alpha
$$

Now the amplitudes of the retained EOFs, $\alpha(L \times M)$, are estimated for each time step $i$ (i.e., each month) by minimizing the optimal interpolation cost function

$$
S\left[\boldsymbol{\alpha}_{:, i}\right]=\left(\mathbf{H}_{i} \mathbf{E} \boldsymbol{\alpha}_{:, i}-\mathbf{T}_{:, i}^{\circ}\right)^{\top} \mathbf{R}_{i}^{-1}\left(\mathbf{H}_{i} \mathbf{E} \boldsymbol{\alpha}_{:, i}-\mathbf{T}_{:, i}^{\circ}\right)+\boldsymbol{\alpha}_{:, i}^{\top} \boldsymbol{\Lambda}^{-1} \boldsymbol{\alpha}_{:, i}
$$

where the notation $\mathbf{A}_{i, i}$ denotes the ith column vector of the matrix $\mathbf{A}$, and $\mathbf{H}_{i}$ is an $N \times n$ matrix containing only $1 \mathrm{~s}$ and 0 s that samples the grid points of the field $\mathbf{T}$ where tide-gauge observations are available at 
time $i$. Note that the tide-gauge distribution may vary from 1 month to another, and hence $\mathbf{H}_{i}$ (and also $N$ ) will vary accordingly. Further, $\mathbf{R}_{i}$ is an $N \times N$ matrix that consists of the sum of the observational $\left(\mathbf{R}_{\mathrm{o}}\right)$ and truncation $\left(\mathbf{H}_{i} \mathbf{E}^{\prime} \Lambda^{\prime} \mathbf{E}^{\prime \top} \mathbf{H}_{i}^{\top}\right)$ error covariance matrices at time $i$ :

$$
\mathbf{R}_{i}=\mathbf{R}_{\mathrm{o}}+\mathbf{H}_{i} \mathbf{E}^{\prime} \Lambda^{\prime} \mathbf{E}^{\top \top} \mathbf{H}_{i}^{\top}
$$

where here $\mathbf{R}_{\mathrm{o}}$ is a diagonal matrix of order $N$.

Finally, minimizing the cost function $S$ gives the optimal interpolation solution

$$
\boldsymbol{\alpha}_{:, i}=\mathbf{P}_{i} \mathbf{E}^{\top} \mathbf{H}_{i}^{\top} \mathbf{R}_{i}^{-1} \mathbf{T}_{:, i}^{\circ}
$$

with $\mathbf{P}_{i}=\left(\boldsymbol{\Lambda}^{-1}+\mathbf{E}^{\top} \mathbf{H}_{i}^{\top} \mathbf{R}_{i}^{-1} \mathbf{H}_{i} \mathbf{E}\right)^{-1}$.

\section{Acknowledgments}

F. M. Calafat was supported under a Marie Curie International Outgoing Fellowship (IOF) within the 7th European Community Framework Programme (grant agreement PIOF-GA-2010-275851). D. P. Chambers was supported under a grant from the NASA Interdisciplinary Science Sponsored Research program. This research would not have been possible without the tide-gauge records from the Permanent Service for Mean Sea Level, housed at the National Oceanography Centre, Liverpool, UK. The authors wish to thank the three anonymous reviewers for their constructive comments on an earlier version of the manuscript.

\section{References}

Allan, R. J., and T. J. Ansell (2006), A new globally complete monthly historical mean sea level pressure data set (HadSLP2): 1850-2004, J. Clim., 19, 5816-5842, doi:10.1175/JCLI3937.1.

Berge-Nguyen, M., A. Cazenave, A. Lombard, W. Llovel, J. Viarre, and J. F. Cretaux (2008), Reconstruction of past decades sea level using thermosteric sea level, tide gauge, satellite altimetry and ocean reanalysis data, Global Planet. Change, 62, 1-13.

Bingham, R. J., and C. W. Hughes (2012), Local diagnostics to estimate density-induced sea level variations over topography and along coastlines, J. Geophys. Res., 117, C01013, doi:10.1029/2011JC007276.

Boyer, T. P., et al. (2009), World Ocean Database 2009, NOAA Atlas NESDIS, vol. 66, edited by S. Levitus, 216 pp., U.S. Gov. Print. Off., Washington, D. C.

Calafat, F. M., and D. Gomis (2009), Reconstruction of Mediterranean sea level fields for the period 1945-2000, Global Planet. Change, 66, $225-234$.

Calafat, F. M., and G. Jordà (2011), A Mediterranean sea level reconstruction (1950-2008) with error budget estimates, Global Planet. Change, 79, 118-133.

Calafat, F. M., D. P. Chambers, and M. N. Tsimplis (2012), Mechanisms of decadal sea level variability in the eastern North Atlantic and the Mediterranean Sea, J. Geophys. Res., 117, C09022, doi:10.1029/2012JC008285.

Calafat, F. M., D. P. Chambers, and M. N. Tsimplis (2013), Inter-annual to decadal sea-level variability in the coastal zones of the Norwegian and Siberian Seas: The role of atmospheric forcing, J. Geophys. Res. Oceans, 118, 1287-1301, doi:10.1002/jgrc.20106.

Carton, J. A., and B. S. Giese (2008), A reanalysis of ocean climate using Simple Ocean Data Assimilation (SODA), Mon. Weather Rev., 136, 2999-3017.

Cazenave, A., and W. Llovel (2010), Contemporary sea level rise, Annu. Rev. Mar. Sci., 2(1), 145-173.

Cazenave, A., O. Henry, S. Munier, T. Delcroix, A. L. Gordon, B. Meyssignac, W. Llovel, H. Palanisamy, and M. Becker (2012), Estimating ENSO Influence on the Global Mean Sea Level, 1993-2010, Mar. Geod., 35, 82-97.

Chambers, D. P., C. A. Mehlhaff, T. J. Urban, D. Fujii, and R. S. Nerem (2002), Low-frequency variations in global mean sea level: 1950-2000, J. Geophys. Res., 107(C4), 3026, doi:10.1029/2001JC001089.

Chambers, D. P., M. A. Merrifield, and R. S. Nerem (2012), Is there a 60-year oscillation in global mean sea level?, Geophys. Res. Lett., 39 , L18607, doi:10.1029/2012GL052885.

Christiansen, B., and F. C. Ljungqvist (2012), Reply to "Comments on 'Reconstruction of the extratropical NH mean temperature over the last millennium with a method that preserves low-frequency variability,"' J. Clim., 25, 7998-8003.

Christiansen, B., T. Schmith, and P. Thejll (2009), A surrogate ensemble study of climate reconstruction methods: Stochasticity and robustness, J. Clim., 22, 951-976.

Christiansen, B., T. Schmith, and P. Thejll (2010), A surrogate ensemble study of sea level reconstructions, J. Clim., 23, 4306-4326, doi: 10.1175/2010JCLI3014.1.

Church, J. A., and N. J. White (2011), Sea-level rise from the late 19th to the early 21st Century, Surv. Geophys., 32, 585-602, doi:10.1007/ s10712-011-9119-1.

Church, J. A., N. J. White, R. Coleman, K. Lambeck, and J. X. Mitrovika (2004), Estimates of the regional distribution of sea level rise over the 1950-2000 period, J. Clim., 17, 2609-2625.

Compo, G. P., et al. (2011), The twentieth century reanalysis project, Q. J. R. Meteorol. Soc., 137, 1-28, doi:10.1002/qj.776.

Dangendorf, S., C. Mudersbach, T. Wahl, and J. Jensen (2013), Characteristics of intra-, inter-annual and decadal sea level variability and the role of meteorological forcing: The long record of Cuxhaven, Ocean Dyn., 63, 209-224.

Enfield, D. B., A. M. Mestas-Nunez, and P. J. Trimble (2001), The Atlantic Multidecadal Oscillation and its relationship to rainfall and river flows in the continental U.S., Geophys. Res. Lett., 28, 2077-2080.

Fan, Y., and H. van den Dool (2004), Climate Prediction Center global monthly soil moisture data set at $0.5^{\circ}$ resolution for 1948 to present, J. Geophys. Res., 109, D10102, doi:10.1029/2003JD004345.

Firing, Y. L., M. A. Merrifield, T. A. Schroeder, and B. Qiu (2004), Interdecadal sea level fluctuations at Hawaii, J. Phys. Oceanogr., 34, 25142524, doi:10.1175/JPO2636.1.

Giese, B. S., and S. Ray (2011), El Niño variability in simple ocean data assimilation (SODA), 1871-2008, J. Geophys. Res., 116, C02024, doi: 10.1029/2010JC006695.

Greatbatch, R. J. (1994), A note on the representation of steric sea level in models that conserve volume rather than mass, J. Geophys. Res., $99,12,767-12,771$.

Hamlington, B. D., R. R. Leben, R. S. Nerem, W. Han, and K.-Y. Kim (2011), Reconstructing sea level using cyclostationary empirical orthogonal functions, J. Geophys. Res., 116, C12015, doi:10.1029/2011JC007529. 
Hamlington, B. D., R. R. Leben, and K.-Y. Kim (2012), Improving sea level reconstructions using non-sea level measurements, J. Geophys. Res., 117, C10025, doi:10.1029/2012JC008277.

Hoerl, A. E., and R. W. Kennard (1970), Ridge regression: An application to non orthogonal problems, Technometrics, 12, 69-82.

Jevrejeva, S., J. C. Moore, A. Grinsted, and P. L. Woodworth (2008), Recent global sea level acceleration started over 200 years ago?, Geophys. Res. Lett., 35, L08715, doi:10.1029/2008GL033611.

Kaplan, A., Y. Kushnir, M. A. Cane, and M. B. Blumenthal (1997), Reduced space optimal analysis for historical data sets: 136 years of Atlantic sea surface temperatures, J. Geophys. Res., 102, 27,835-27,860.

Kaplan, A., Y. Kushnir, and M. A. Cane (2000), Reduced space optimal interpolation of historical marine sea level pressure, J. Clim., 13, 29873002.

Llovel, W., A. Cazenave, P. Rogel, A. Lombard, and M. B. Nguyen (2009), Two-dimensional reconstruction of past sea level (1950-2003) from tide gauge data and an Ocean General Circulation Model, Clim. Past, 5, 217-227, doi:10.5194/cp-5-217-2009.

Llovel, W., M. Becker, A. Cazenave, S. Jevrejeva, R. Alkama, B. Decharme, H. Douville, M. Ablain, and B. Beckley (2011), Terrestrial waters and sea level variations on interannual time scale, Global Planet. Change, 75, 76-82, doi:10.1016/j.gloplacha.2010.10.008.

Marcos, M., and M. N. Tsimplis (2007), Variations of the seasonal sea level cycle in southern Europe, J. Geophys. Res., 112, C12011, doi: 10.1029/2006JC004049.

Marcos, M., M. N. Tsimplis, and F. M. Calafat (2012), Inter-annual and decadal sea level variations in the north-western Pacific marginal seas, Prog. Oceanogr., 105, 4-21.

McLean, N. M., J. F. Bowring, and S. A. Bowring (2011), An algorithm for U-Pb isotope dilution data reduction and uncertainty propagation, Geochem. Geophys. Geosyst., 12, Q0AA18, doi:10.1029/2010GC003478.

Merrifield, M. A. (2011), A shift in western tropical Pacific sea-level trends during the 1990s, J. Clim., 24, 4126-4138, doi:10.1175/ 2011JCLI3932.1.

Meyssignac, B., M. Becker, W. Llovel, and A. Cazenave (2012), An assessment of two-dimensional past sea level reconstructions over 19502009 based on tide-gauge data and different input sea level grids, Surv. Geophys., 33, 945-972.

Miller, L., and B. C. Douglas (2007), Gyre-scale atmospheric pressure variations and their relation to 19th and 20th century sea level rise, Geophys. Res. Lett., 34, L16602, doi:10.1029/2007GL030862.

Miller, S. K. (1981), On the inverse of the sum of matrices, Math. Mag., 54, 67-72.

Nerem, R.S., D. P. Chambers, C. Choe, and G. T. Mitchum (2010), Estimating mean sea level change from the TOPEX and Jason altimeter missions, Mar. Geod., 33, 435-446.

Peltier, W. R. (2004), Global glacial isostasy and the surface of the ice-age earth: The ICE-5G (VM2) model and GRACE, Ann. Rev. Earth Planet. Sci., 32, 111-149.

Ponte, R. M. (1999), A preliminary model study of the large-scale seasonal cycle in bottom pressure over the global ocean, J. Geophys. Res., 104, 1289-1300.

Prichard, D., and J. Theiler (1994), Generating surrogate data for time series with several simultaneously measured variables, Phys. Rev. Lett., 73, 951-954.

Ray, R., and B. Douglas (2011), Experiments in reconstructing twentieth-century sea levels, Prog. Oceanogr., 91, 496-515.

Riva, R. E. M., J. L. Bamber, D. A. Lavallée, and B. Wouters (2010), Sea-level fingerprint of continental water and ice mass change from GRACE, Geophys. Res. Lett., 37, L19605, doi:10.1029/2010GL044770.

Smerdon, J. E. (2012), Climate models as a test bed for climate reconstruction methods: Pseudoproxy experiments, WIREs Clim. Change, 3, 63-77. doi:10.1002/wcc.149.

Vinogradov, S. V., and R. M. Ponte (2010), Annual cycle in coastal sea level from tide gauges and altimetry, J. Geophys. Res., 115, C04021, doi:10.1029/2009JC005767.

Wahl, T., I. Haigh, P. L. Woodworth, F. Albrecht, D. Dillingh, J. Jensen, R. Nicholls, R. Weisse, and G. Wöppelmann (2013), Observed mean sea level changes around the North Sea coastline from 1800 to present, Earth Sci. Rev., 124, 51-67.

Wolter, K., and M. S. Timlin (1998), Measuring the strength of ENSO events: How does 1997/98 rank?, Weather, 53, 315-324.

Wolter, K., and M. S. Timlin (2011), El Niño/Southern Oscillation behaviour since 1871 as diagnosed in an extended multivariate ENSO index (MEl.ext), Int. J. Climatol., 31, 1074-1087.

Woodworth, P. L., and R. Player (2003), The permanent service for mean sea level: An update to the 21 st century, J. Coastal Res., 19, 287295. 